\title{
Calibration of 3-D wind measurements on a single-engine research aircraft
}

\author{
C. Mallaun ${ }^{1}$, A. Giez ${ }^{1}$, and R. Baumann ${ }^{2}$ \\ ${ }^{1}$ Deutsches Zentrum für Luft- und Raumfahrt (DLR), Flight Experiments, Oberpfaffenhofen, Germany \\ ${ }^{2}$ Deutsches Zentrum für Luft- und Raumfahrt (DLR), Institute of Atmospheric Physics, Oberpfaffenhofen, Germany \\ Correspondence to: C. Mallaun (christian.mallaun@dlr.de)
}

Received: 18 December 2014 - Published in Atmos. Meas. Tech. Discuss.: 11 February 2015

Revised: 15 June 2015 - Accepted: 22 July 2015 - Published: 12 August 2015

\begin{abstract}
An innovative calibration method for the wind speed measurement using a boom-mounted Rosemount model $858 \mathrm{AJ}$ air velocity probe is introduced. The method is demonstrated for a sensor system installed on a mediumsize research aircraft which is used for measurements in the atmospheric boundary layer. The method encounters a series of coordinated flight manoeuvres to directly estimate the aerodynamic influences on the probe and to calculate the measurement uncertainties. The introduction of a differential Global Positioning System (DGPS) combined with a highaccuracy inertial reference system (IRS) has brought major advances to airborne measurement techniques. The exact determination of geometrical height allows the use of the pressure signal as an independent parameter. Furthermore, the exact height information and the stepwise calibration process lead to maximum accuracy. The results show a measurement uncertainty for the aerodynamic influence of the dynamic and static pressures of $0.1 \mathrm{hPa}$. The applied parametrisation does not require any height dependencies or time shifts. After extensive flight tests a correction for the flow angles (attack and sideslip angles) was found, which is necessary for a successful wind calculation. A new method is demonstrated to correct for the aerodynamic influence on the sideslip angle. For the three-dimensional (3-D) wind vector (with $100 \mathrm{~Hz}$ resolution) a novel error propagation scheme is tested, which determines the measurement uncertainties to be $0.3 \mathrm{~m} \mathrm{~s}^{-1}$ for the horizontal and $0.2 \mathrm{~m} \mathrm{~s}^{-1}$ for the vertical wind components.
\end{abstract}

\section{Introduction}

The three-dimensional (3-D) wind vector from an aircraft is measured as the difference between the ground speed ( $g s$ ) and the true airspeed (tas) vectors as sketched in Fig. 1a. The former vector describes the motion of the aircraft relative to the ground and the latter relative to the air. Any error in both of these velocities impacts directly the wind calculation. As the wind components are often 1 magnitude of size smaller than the aircraft velocities, the accuracy of these speed measurements is critical. The magnitude of the tas is usually calculated from the static and dynamic pressure; the direction is defined by the angles of attack and sideslip. Measuring these quantities with a gust probe (or similar instrumentation) near the aircraft is hindered by the inevitable flow distortion caused by the aircraft itself (e.g. compression, upwash). Even with a long boom it is not possible to position the probe in the undisturbed flow and therefore a set of well-designed test flights is needed to parametrise the static pressure error and the flow angle deflection (Bange et al., 2013).

Traditionally the basic calibration starts with the determination of the static pressure error (Gracey, 1979), which is the deviation of the pressure measured at the static pressure port from the undisturbed air pressure. To complete the calibration a series of calibration manoeuvres such as reverse headings, speed variations, steady sideslips, and pitch-andyaw oscillations are performed to calculate the correct flow angles and dynamic dependencies of the measurement system (e.g. Boegel and Baumann, 1991; Lenschow and SpyersDuran, 1989; Tjernstroem and Friehe, 1991; Khelif et al., 1999). Rodi and Leon (2012) describe a method to directly calculate the flow angles, dynamic pressure and static pressure error solving a set of equations that follows from the 

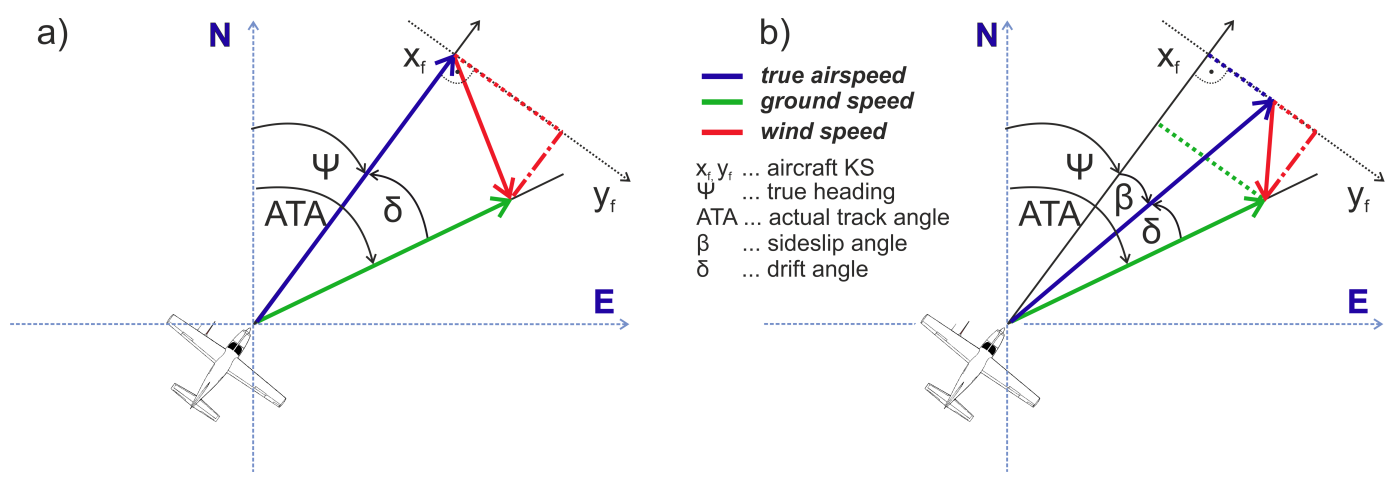

Figure 1. (a) Formation of the wind triangle (two-dimensional) which is used to calculate the wind from an aircraft. (b) Expanded wind triangle; the figure includes the sideslip angle $\beta$. This angle is nonzero when for example the aircraft is not flying straight ahead relative to the aircraft coordinate system, the reference system is not well aligned or turbulent wind fluctuations are present.

predicted variation of the pressure on the hemispherically shaped gust probe. They could achieve a precision for the pressure correction of better than $\pm 20 \mathrm{~Pa}$ for a wide range of speeds and aircraft configurations. The key to these small errors is the implementation of a differential Global Positioning System (DGPS) combined with a high-accuracy inertial reference system (IRS). It enables the exact estimation of the geometric height resolving all the fluctuations due to turbulence or small attitude changes of the aircraft, and thus the height influence on the pressure calibration can effectively be reduced. Up to now, difficulties in the estimation of the gs with the onboard avionic system have usually been the major source of errors in the wind calculation (Boegel and Baumann, 1991), which is overcome by the implementation of the combined IRS and DGPS.

This new technology is available for a meteorological sensor package on a Cessna Grand Caravan 208B (Caravan). The IRS is installed within the cabin, while the gust probe is mounted on a $2 \mathrm{~m}$ boom under the left wing of the aircraft. We describe the calibration of this system as an example of how to fulfill the entire calibration procedure in order to calculate the 3-D wind. Details about the wind calculation on an aircraft are described in the following section including a very robust method for how the angular difference between the gust probe and the IRS can be estimated and corrected. In Sect. 3 we introduce the measurement system on the Caravan and describe in Sect. 4 a stepwise method to complete the calibration of the implemented pitot-static system. The influences of the flow distortion on the involved units are corrected one after the other, which brings two major advantages: it allows an instructive insight into the details and impacts of the different processes and allows for a straightforward calculation of the residual errors. The study is completed with detailed considerations about the resulting errors in the 3-D wind calculation and final conclusions.

\section{Wind calculation}

To calculate the 3-D wind vector $(\boldsymbol{v})$,

$v=g s-t a s+\Omega \times L$

has to be solved. The third term on the right-hand side of Eq. (1) accounts for the motion from the aircraft angular velocities $(\boldsymbol{\Omega})$ and the lever arm $(\boldsymbol{L})$, which represents the distance between the gust probe and the IRS.

$\boldsymbol{L}$ is a constant aircraft property measured on the ground; $\boldsymbol{\Omega}$ and the $\boldsymbol{g} \boldsymbol{s}$ are direct outputs of the IRS. Following Bernoulli's theorem for a compressible gas, the TAS is calculated using

$\mathrm{TAS}=\sqrt{2 \cdot\left(\frac{k}{k-1}\right) \cdot R \cdot T_{\mathrm{S}} \cdot\left[\left(1+\frac{q_{\mathrm{c}}}{p_{\mathrm{s}}}\right)^{\frac{k-1}{k}}-1\right]}$

with the adiabatic index $k$ and the gas constant $R$ for humid air (Bange et al., 2013). For this calculation the dynamic pressure $\left(q_{\mathrm{c}}\right)$, the static pressure $\left(p_{\mathrm{s}}\right)$ and the temperature $\left(T_{\mathrm{s}}\right)$ are required. The flow angles (i.e. the attack angle $(\alpha)$ and sideslip angle $(\beta))$ define the direction of the TAS relative to the aircraft (index $f$ ) using

$\boldsymbol{t a s}_{\mathrm{f}}=\frac{\mathrm{TAS}}{D} \cdot\left(\begin{array}{c}1 \\ \tan (\beta) \\ \tan (\alpha)\end{array}\right)$,
$D=\sqrt{1+\tan ^{2}(\alpha)+\tan ^{2}(\beta)}$

(Lenschow and Spyers-Duran, 1989). To gain high-quality measurements of the pressure signals and the flow angles, flight test equipment such as a boom-mounted gust probe have to be installed on the aircraft.

While the wind and the $\boldsymbol{g} \boldsymbol{s}$ are usually measured in an Earth fixed coordinate system (CS) ( $x$ axis north, $y$ axis east, $z$ axis downward), the $\boldsymbol{t a s}$ and $\boldsymbol{\Omega}$ are available in the aircraft fixed CS (an orthogonal CS with the $x$ axis along the centre of the fuselage positive to the front, $y$ axis positive to the 
right and $z$ axis positive downward) and therefore have to be rotated before solving Eq. (1). Finally, the components of $v$ need to be resorted in order to get the 3-D wind in the meteorological CS so that the first component $(u)$ is positive to the east, the second $(v)$ to the north and the vertical wind $(w)$ positive during updrafts. Lenschow and Spyers-Duran (1989) describe the methodology for rotating aircraft to Earth coordinate axes where they also describe the involved parameters and CSs. Lenschow (1986) simplified the following set of equations in level flight to be

$u \approx-\mathrm{TAS} \cdot \sin (\Psi+\beta)+v_{\mathrm{ew}}$,

$v \approx-\mathrm{TAS} \cdot \cos (\Psi+\beta)+v_{\mathrm{ns}}$,

$w \approx-\mathrm{TAS} \cdot \sin (\Theta-\alpha)+v_{\mathrm{v}}$,

where the $\boldsymbol{g} \boldsymbol{s}$ is split into its components the east-west velocity $\left(v_{\text {ew }}\right)$, the north-south velocity $\left(v_{\text {ns }}\right)$, and the vertical velocity $\left(v_{\mathrm{V}}\right)$. They give an estimation of the first-order terms during straight horizontal flight. One has to take care with the rotation of $\boldsymbol{\Omega} \times \boldsymbol{L}$. The systems we are using provide all three components of $\boldsymbol{\Omega}$ in the aircraft fixed CS, which are the angular velocities from the rotation along the three axes of the CS. Thus, the full rotation with the three attitude angles (roll angle $(\Phi)$, pitch angle $(\Theta)$ and heading angle $(\psi))$ must be performed in order to transform the vector into the Earth fixed CS. On the other hand Lenschow and Spyers-Duran (1989) describe a system where the angular velocities are defined as the time derivatives of the three rotation angles $(\dot{\Phi}, \dot{\Theta}, \dot{\Psi})$. In this case the calculation of $\boldsymbol{\Omega} \times \boldsymbol{L}$ and the rotation have to be performed stepwise corresponding to the respective rotation angle. Especially during steep turns the usage of the correct method is essential in order to avoid significant errors.

\subsection{Considerations about the wind measurement}

The principle of the wind triangle is visualised for the twodimensional case in Fig. 1. Without wind the $\boldsymbol{g} s$ and the $\boldsymbol{t a s}$ are equal. Any wind component along the flight direction changes the magnitude of the $\boldsymbol{g} \boldsymbol{s}$, while the $\boldsymbol{t a s}$ and the direction of the vectors remain almost the same. On the other hand, a cross-wind component changes primarily the direction of the $\boldsymbol{g} \boldsymbol{s}$, because it shifts the aircraft with respect to the ground. The drift angle $(\delta)$ is the difference between the true heading $(\Psi)$ - the direction the nose of the aircraft points to relative to north - and the actual track angle (ATA) - the direction the aircraft moves relative to the Earth fixed CS. $\delta$ determines the strength of the cross-wind component, while the wind component along the aircraft results primarily from the difference between the respective ground speed component and the TAS.

In this idealised case the nose of the aircraft always points in the direction of the airflow (i.e. along the tas) and with this the sideslip angle $(\beta)$ is zero. In reality different effects such as small deviations from the aircraft symmetry, bad trimming or a misalignment of the IRS with respect to the aircraft CS result in a nonzero $\beta$ which has to be included in the wind calculation. Figure 1b shows an expanded concept where $\delta$ must be corrected for the value of $\beta$. This concept becomes very important when a high-frequency wind (e.g. $>1 \mathrm{~Hz})$ is measured. The aircraft tends to align with the tas, but because of inertia it is not fast enough to follow the turbulent fluctuations. The bigger and heavier the aircraft, the slower it usually reacts to changes in the wind signal. The magnitude of $\beta$ fluctuations corresponds to the strength of turbulence and inertia of the aircraft. On the other hand, a non-zero mean beta angle - on timescales of more than a few seconds - indicates a non-symmetry of the air flow at the location of the probe and/or misalignment between the main axes of the aircraft, five-hole probe, and inertial reference system. Therefore, such systematic angular offsets have to be determined carefully for high-precision wind calculation.

Similar to $\beta$, the turbulent fluctuations of the vertical wind appear in the attack angle $(\alpha)$ which is used to calculate the vertical component of the tas. Again, the aircraft is responding slower to the fluctuations, which is why they do not appear in the vertical aircraft velocity. Even though the vertical wind fluctuations can reach values of several metres per second $\left(\mathrm{m} \mathrm{s}^{-1}\right)$, the mean vertical wind is usually small (Liljequist and Cehak, 1984). Averaging over a long enough time period (e.g. a complete flight), the vertical wind will vanish due to mass continuity. As usually an aircraft navigation system does not measure the flow angles, it cannot measure the vertical wind and for the calculation of the horizontal wind it has to assume $\beta=0$, which leads to errors.

\subsection{Calculation of the flow angle offsets $\varepsilon_{\mathrm{b}}$ and $\eta_{\mathrm{b}}$}

The attack and sideslip angles are often measured with a gust probe, which might have a significant tilt relative to the aircraft CS. Also, for the IRS small deviations from the aircraft CS will be unavoidable, even though it is usually carefully aligned with the aircraft (e.g. the seat rail in the cabin can be the reference). Lenschow and Spyers-Duran (1989) demonstrated the sensitivity of the flow angles in the wind calculation. A change of $0.1^{\circ}$ at an airspeed of $100 \mathrm{~m} \mathrm{~s}^{-1}$ is equivalent to a change of $17 \mathrm{~cm} \mathrm{~s}^{-1}$ in the lateral or vertical air velocity. This emphasises the importance of the exact definition of the CSs and the correction of the respective angle offset between them. The determination of the exact orientation of the aircraft CS turns out to be very difficult. However, for the wind calculation according to Eq. (1), this is not necessary. It is sufficient to take the orientation of the IRS as the reference and to estimate the flow angles relative to it. Boegel and Baumann (1991) suggest two correction coefficients $\varepsilon_{\mathrm{b}}$ and $\eta_{\mathrm{b}}$ that are added to the attack angle and to the sideslip angle, respectively. Calculating

$\alpha=\alpha_{\mathrm{NB}}+\varepsilon_{\mathrm{b}} \quad$ and $\quad \beta=\beta_{\mathrm{NB}}+\eta_{\mathrm{b}}$

is a simple and efficient way to correct the flow angles measured with the gust probe mounted on a nose boom (index 
$\mathrm{NB})$ to the reference CS. To calculate these correction coefficients for an individual flight, we suggest a very robust method that is based on two basic conditions:
(i) $\langle w\rangle=0$ and
(ii) $\operatorname{Cov}(w, \sin (\Phi))=0$,

for long enough flight periods where $\operatorname{Cov}()$ represents the covariance of the embraced parameters (e.g. Wilks, 2006). Condition (i) says that for the entire flight the mean vertical wind $\langle w\rangle$ vanishes. Following the discussion in Sect. 2.1 the mean vertical wind is very small when the averaging period is long enough. Assuming that the other involved parameters are well calibrated, a mean offset of $\alpha$ produces an offset in $\langle w\rangle$. Thus, this condition is applied to calculate the attack angle offset $\left(\varepsilon_{\mathrm{b}}\right)$. It is the same condition as used by Khelif et al. (1999) in Sect. 5 for the calibration of the attack angle offset for which we suggest an analytical solution.

The beta-offset $\left(\eta_{\mathrm{b}}\right)$ calculation is based on condition (ii), which states that during turns no correlation between roll angle $(\Phi)$ and the vertical wind is allowed. This is true, because the vertical wind must be independent of the attitude angles of the aircraft. As long as there exists a constant offset in the sideslip angle beta (i.e. the boom is tilted either to the left or right) any rotation around the $x$ axis (e.g. during turns) effectively tilts up or down the boom. This vertical tilt produces an artificial signal in the vertical wind, which is the product of the sine of the roll angle times the beta-offset $\eta_{\mathrm{b}}$ times the true airspeed (i.e. $\mathrm{dw} \sim \eta_{\mathrm{b}} \cdot \sin (\Phi)$. TAS). The sign of the "wrong" vertical wind (dw) changes according to whether it is a left turn or a right turn, and so the effect of $\eta_{\mathrm{b}}$ can be discriminated from an attack angle offset $\left(\varepsilon_{\mathrm{b}}\right)$, because the latter does not depend on the direction of the turn. A wrong $\varepsilon_{\mathrm{b}}$ does not influence the result of $\eta_{\mathrm{b}}$. Note that the method is meant to correct for a pure geometric (mechanical) misalignment between the two coordinate systems of the 5HP and the IRS, which can be assumed to be independent of flight state. It cannot replace the pressure and flow angle calibration described in Sect. 4, but is an additional step in order to optimise the wind calculation. Just the periods during turns (e.g. $|\Phi|>10^{\circ}$ ) are taken for the calculation of $\eta_{\mathrm{b}}$, because then the sideslip angle has a significant contribution to the vertical wind component (e.g. $w \sim \sin \beta \cdot \sin \Phi \cdot \cos \Theta$ ). On the other hand, condition (i) is applied for the periods when the aircraft flies straight ahead to avoid any negative influence of $\beta$. Thus, two independent data sets are available for each individual flight to calculate the flow angle offsets $\varepsilon_{\mathrm{b}}$ and $\eta_{\mathrm{b}}$.

Starting with an appropriate initial guess for $\varepsilon_{\mathrm{b}}$ and $\eta_{\mathrm{b}}$ in Eq. (5) a biased vertical wind $\left(w_{\text {old }}\right)$ can be calculated. The difference between $w_{\text {old }}$ and the corrected vertical wind $\left(w_{\text {new }}\right)$ is defined by

$w_{\text {new }}=w_{\text {old }}+\frac{\partial w}{\partial \varepsilon_{\mathbf{b}}} \cdot \mathrm{d} \varepsilon_{\mathbf{b}}+\frac{\partial w}{\partial \eta_{\mathrm{b}}} \cdot \mathrm{d} \eta_{\mathrm{b}}$.

The partial derivatives in the equation can be directly calculated from the third component of the wind equation (e.g.
Lenschow and Spyers-Duran, 1989) (see Appendix). $w_{\text {new }}$ must fulfill the conditions in Eq. (6). Thus, we can substitute the right side of Eq. (7) into condition (i) of Eq. (6), which leads to

$\mathrm{d} \varepsilon_{\mathrm{b}}=-\frac{\left\langle w_{\text {old }}\right\rangle}{\left\langle\partial w / \partial \varepsilon_{\mathbf{b}}\right\rangle}$,

assuming that $\left\langle\partial w / \partial \eta_{\mathrm{b}}\right\rangle \ll\left\langle\partial w / \partial \varepsilon_{\mathrm{b}}\right\rangle$, which is true for small roll angles. For condition (ii) of Eq. (6) we obtain

$\mathrm{d} \eta_{\mathrm{b}}=-\frac{\operatorname{Cov}\left(w_{\text {old }}, \sin (\Phi)\right)}{\operatorname{Cov}\left(\frac{\partial w}{\partial \eta_{\mathrm{b}}}, \sin (\Phi)\right)}$,

taking into account that $\operatorname{Cov}\left(\frac{\partial w}{\partial \varepsilon_{\mathrm{b}}}, \sin (\Phi)\right) \approx 0$ (see Appendix).

The results are used to calculate the correct offset angles $\varepsilon_{\mathrm{b}}$ and $\eta_{\mathrm{b}}$ with

$\varepsilon_{\mathrm{b}}=\varepsilon_{\mathrm{b}}($ old $)+\mathrm{d} \varepsilon_{\mathrm{b}} \quad$ and $\quad \eta_{\mathrm{b}}=\eta_{\mathrm{b}}($ old $)+\mathrm{d} \eta_{\mathrm{b}}$.

These values are needed to calculate the correct flow angles and finally also the correct 3-D wind signal.

In the programming code the method requires several steps: to start the procedure, the vertical wind $\left(w_{\text {old }}\right)$ is calculated using first guess values of $\varepsilon_{\mathrm{b}}$ (old) and $\eta_{\mathrm{b}}$ (old) (e.g. the values from the preceding flight). In the second step, Eqs. (9) and (10) are used to correct for the sideslip offset. The third step is to calculate an improved vertical wind with the new $\beta$ from Eq. (5). In step 4 the attack angle offset is corrected using Eqs. (8) and (10). Finally the correct values for $\varepsilon_{\mathrm{b}}$ and $\eta_{\mathrm{b}}$ are used in Eq. (5) to obtain the correct attack and sideslip angle with respect to IRS, which defines the reference CS. These steps can be repeated iteratively to gain optimum results. Usually already the second iteration does not add any significant changes.

It is important that the calculation is performed for the entire flight, which has to be long enough and must include several turns to get reliable results. A bias in the correction values is also possible when the aircraft flies systematically in updraft or downdraft regions. Such biases will be discovered easily by comparing the result with previous flights, which is a necessary step in the quality control. The results of this calculation for more than 800 research flights with a Dassault Falcon20 since 2001 are displayed in Fig. 2 (note that for statistical reasons we show the results from a different aircraft here; for the new measurement system on the Caravan, far fewer flights are available). The variation of $\varepsilon_{\mathrm{b}}$ is small compared to the results of $\eta_{\mathrm{b}}$, which has several reasons. For the calculation of $\varepsilon_{\mathrm{b}}$ more data are available (i.e. a flight usually consists of more straight flight legs than turns) and the calculation method is more simple. In contrast to $\varepsilon_{\mathrm{b}}, \eta_{\mathrm{b}}$ is calculated during non-steady flight conditions, which generates an indeterminable influence by aircraft dynamics. The 


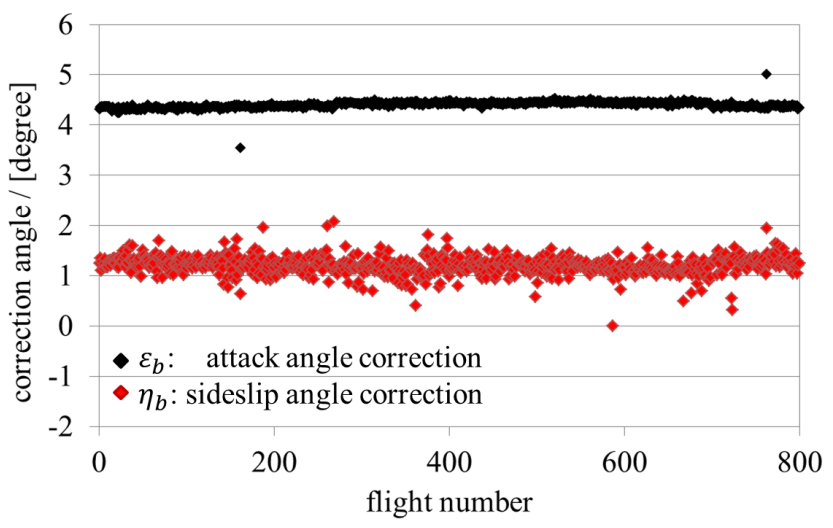

Figure 2. Results of the flow angle correction for 803 flights with the DLR Falcon20 research aircraft after 2001. The correction for the attack angle offset $(\alpha)$ shows significantly less scatter than for the sideslip angle $(\beta)$. The offset of $\alpha \sim 4^{\circ}$ corresponds to a mean attack angle during normal flight conditions. The nose boom on the aircraft is tilted downward about this angle to achieve a straight flow towards the gust probe.

nose boom and the IRS are permanently installed on the Falcon20, which avoids any discontinuities in the data. However, a very slow drift over the years in the attack angle correction is visible. The two points with a significant offset in the data (flights 162 and 763) indicate an obvious defect in the measurement system. In such a case the estimation of $\varepsilon_{\mathrm{b}}$ and $\eta_{\mathrm{b}}$ for this flight is not valid.

It is not possible to reach a similar accuracy for the flow angles through a direct measurement of the nose boom and IRS alignment on the ground. Additionally to the technical difficulties, no ground-based procedure is available to account for the aero-dynamical effects (e.g. compression, flow distortion) significantly influencing the result as well.

\section{The research aircraft: Cessna Grand Caravan 208B}

The research aircraft used for this study is a modified Cessna Grand Caravan 208B (see Fig. 3). The robust and efficient aircraft is equipped with a powerful single-engine turboprop that guarantees a high manoeuvrability and climb performance. Some of the key properties of the aircraft are listed in Table 1. The basic modifications of the aircraft include several openings in the unpressurised fuselage, large apertures in the rear of the cabin and an autonomous experimental power system $(200 \mathrm{~A}$ at $28 \mathrm{~V})$. Underwing hard points are provided to support measurement containers from which the left one is used for the meteorological sensor package (METPOD) carrying the $2 \mathrm{~m}$ long nose boom as shown in Fig. 3.

\subsection{Meteorological measurement equipment}

The basic meteorological measurement equipment in the research aircraft consists of three major elements: (i) the MET-

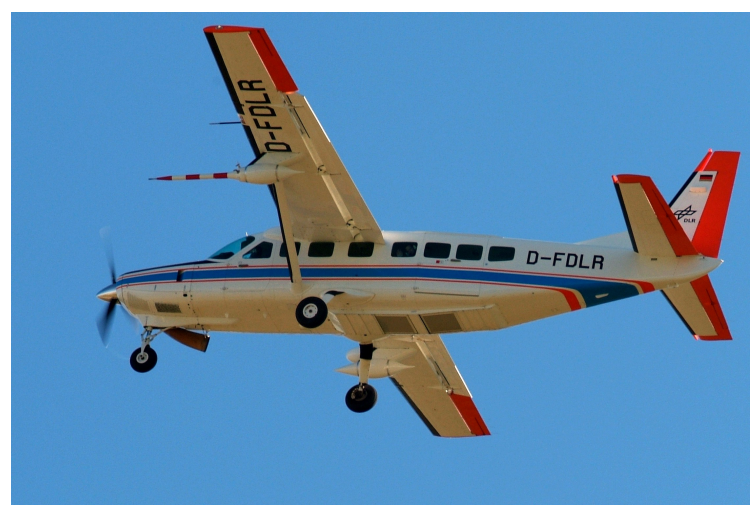

Figure 3. Cessna Grand Caravan 208B with meteorological sensor package (METPOD) mounted under the left wing.

Table 1. Key properties of the research aircraft.

\begin{tabular}{lr}
\hline \multicolumn{2}{l}{ Cessna Grand Caravan 208B "D-FDLR” } \\
\hline Length & $12.7 \mathrm{~m}$ \\
Max. takeoff weight & $3970 \mathrm{~kg}$ \\
Max. payload with maximum fuel & $500 \mathrm{~kg}$ \\
Max. altitude (ISA) & $7600 \mathrm{~m}$ \\
Max. range & $1950 \mathrm{~km}$ \\
Max. endurance & $7 \mathrm{~h}$ \\
\hline
\end{tabular}

POD containing the main sensors for pressure, temperature, humidity and wind measurement, (ii) the AEROcontrol from IGI systems (IGI), which is a combined DGPS and IRS system for high-accuracy measurement of the aircraft position and attitude (Cramer, 2001), (iii) the main data processing unit, "measurement acquisition of meteorological basics" (blackMAMBA), containing a real-time system for data acquisition, a computer providing quicklook data, and a time server. Details of the relevant hardware and sensor components are listed in Table 2. Several measures are taken to guarantee the data quality of the signals acquired in the METPOD (e.g. bonding concept, heating of the container). To compensate for the significant temperature sensitivity of the pressure transducers, they are actively temperature stabilised at $28^{\circ} \mathrm{C}$. The transducers are connected to the fivehole probe (5HP) on the tip of the nose boom with $2 \mathrm{~m}$ long tubes. The small diameter of the hemisphere (e.g. $26 \mathrm{~mm}$ ) and the short distance between dynamic and static pressure ports (e.g. $<100 \mathrm{~mm}$ ) are advantageous for measuring smallscale fluctuations.

The nose boom is tilted downward about $4^{\circ}$ with respect to the aircraft CS to compensate for a mean attack angle during average flight conditions to align the probe along the mean flow direction. The gust probe has a limited angular acceptance range relative to its zero position where the dynamic pressure is unaffected from the flow angle and the differential pressure measures respond linearly to flow angle changes 
Table 2. List of the main hardware components included in the three major parts of the meteorological sensor system on the Caravan.

\begin{tabular}{lcl}
\hline Component & Model & Manufacturer \\
\hline & METPOD & \\
\hline $\begin{array}{l}\text { Five-hole probe } \\
\text { Temperature housing, humidity inlet }\end{array}$ & Model 858AJ & Rosemount \\
Absolute/differential pressure transducer & PMP 4100 & Rosemount \\
Open-wire PT100 & & Druck \\
Temperature conditioner & Model 0510GA & DLR \\
$\begin{array}{l}\text { Capacitive humidity sensor } \\
\text { Dewpoint mirror }\end{array}$ & Humicap HMP230 & Vaisala \\
Ly- $\alpha$ absorption hygrometer & Model TP3-S & Meteolabor, modified by DLR \\
$\begin{array}{l}\text { Three-axis accelerometer } \\
\text { 16-bit analogue/digital device (with CAN output) }\end{array}$ & Model L5 & Buck research \\
\hline & PN 979-1200 & Sundstrand data control \\
& AEROcontrol (IGI) & RD Electronics \\
\hline $\begin{array}{l}\text { Sensor management unit (SMU) } \\
\text { Inertial measurement unit (IMU) }\end{array}$ & Model IId & IGI systems \\
\hline & blackMAMBA & \\
\hline Real-time data acquisition system & PXI-8102 & National Instruments \\
18-bit analogue/digital device & PXI-6284 & National Instruments \\
Embedded PC for real-time data visualisation & Mayflower-eM & Advantech Co. \\
Time server & Lantime M900 & Meinberg \\
\hline
\end{tabular}

(e.g. $\left.\pm 10^{\circ}\right)$. The three independent humidity sensors $(\mathrm{Hu}-$ micap Vaisala HMP230 capacitive sensor (Humicap), Meteolabor TP3-S dewpoint mirror (TP3) and a Buck research model L5 Ly- $\alpha$ absorption hygrometer $(\mathrm{Ly}-\alpha)$ ) are collocated in the humidity channel. Additional sensors for temperature and pressure within the humidity channel are used to correct for the modified thermodynamic conditions at the position of the humidity sensors. The static temperature is measured with two redundant open-wire PT100 sensors mounted with two total air temperature (TAT) housings (e.g. Bange et al., 2013) (Rosemount Model 102B) on the left- and righthand side of the leading cone on the METPOD. The system includes several housekeeping parameters within the METPOD (e.g. sensor voltage, temperature in five different positions, 3-D acceleration, temperature and heating power for each pressure transducer) to monitor the operation of the sensor system. The IGI system consists of an inertial measurement unit (IMU) based on fibre optic gyros and the sensor management unit with an integrated high-end GPS receiver. The GPS data are improved during the flight with a real-time differential correction signal via satellite (OMNISTAR). Already during flight the GPS signal and the information of the IMU are combined to high-precision information of position and attitude. During post-processing the optimum results are achieved with commercially available DGPS correction signals and optimised correction routines including the entire data set of each individual flight. This leads to an extremely high accuracy of the data as listed in Table 3. The blackMAMBA is recording and preparing the collected data for real-time visualisation from the METPOD, the IGI, the onboard flight management system and additional sensors within the cabin (e.g. event marker, accelerometer, rudder position sensor). The time server provides different trigger signals and time sources for synchronisation of the involved data sources. The Quicklook PC calculates the corrected physical parameters and displays them in real time on up to seven operator screens in the cabin. On the dashboard a forward-looking full-HD camera is installed.

\subsection{Sensor calibration and accuracy}

The regular calibration of all the components preserves the quality of the various sensors. The calibration of the IGI is performed by the manufacturer, while the calibration of the pressure, temperature and humidity sensors is performed in-house with test equipment which is traceable to national standards. A list of the involved quantities with their relevant properties is shown in Table 3. Details on the pressure calibration can be found in Mallaun and Giez (2013) demonstrating a measurement uncertainty for all the pressure sensors of less than $\pm 25 \mathrm{~Pa}$.

The first calibration step of the temperature sensors is realised in a stirred fluid bath with a precision reference sensor (Heraeus PW-EZ 100 PRT). Including the second step the calibration of the signal path - the measurement uncertainty of the temperature sensors is calculated to be $\pm 0.05 \mathrm{~K}$ within the range of -70 to $+50^{\circ} \mathrm{C}$. For the overall accuracy of the static temperature, contributions of the analogue de- 
Table 3. List of the main measurement parameters included in the meteorological sensor package on the Caravan. The frequency describes the rate of recording after the appropriate filtering and $\sigma$ is the overall uncertainty of the listed parameters including all errors such as sensor errors, temperature dependencies, analogue conversion or aerodynamic effects. With the in-house calibration equipment the results are traceable to national standards. The uncertainties in AEROcontrol IGI is according to the specifications of the manufacturer (Cramer, 2001). The uncertainties of derived parameters include the errors of all the involved parameters and corrections.

\begin{tabular}{|c|c|c|c|c|c|}
\hline Quantity & Variable & Range & Resolution & Frequency & $\sigma$ \\
\hline \multicolumn{6}{|c|}{ Gust probe } \\
\hline Static pressure & $p_{\mathrm{s}}$ & $0-1200 \mathrm{hPa}$ & $2 \mathrm{~Pa}$ & $100 \mathrm{~Hz}$ & $0.25 \mathrm{hPa}$ \\
\hline Dynamic pressure & $q_{\mathrm{c}}$ & $0-120 \mathrm{hPa}$ & $0.2 \mathrm{~Pa}$ & $100 \mathrm{~Hz}$ & $0.15 \mathrm{hPa}$ \\
\hline Differential pressure $\alpha / \beta$ & $\mathrm{d} p_{\mathrm{a}} / \mathrm{d} p_{\mathrm{b}}$ & $\pm 50 \mathrm{hPa}$ & $0.15 \mathrm{~Pa}$ & $100 \mathrm{~Hz}$ & $0.1 \mathrm{hPa}$ \\
\hline \multicolumn{6}{|c|}{ AEROcontrol IGI } \\
\hline Position & & & & $128 \mathrm{~Hz}$ & $0.05 \mathrm{~m}$ \\
\hline Altitude & alt & & & $128 \mathrm{~Hz}$ & $0.05 \mathrm{~m}$ \\
\hline E-W/N-S vertical velocity & $v_{\mathrm{ew}} / v_{\mathrm{ns}} / v_{\mathrm{V}}$ & & & $128 \mathrm{~Hz}$ & $0.005 \mathrm{~m} \mathrm{~s}^{-1}$ \\
\hline Roll/pitch & $\Phi / \Theta$ & $\pm 90^{\circ}$ & & $128 \mathrm{~Hz}$ & $0.004^{\circ}$ \\
\hline Heading & $\Psi$ & $0-360^{\circ}$ & & $128 \mathrm{~Hz}$ & $0.01^{\circ}$ \\
\hline \multicolumn{6}{|c|}{ Temperature } \\
\hline Total air temperature 1 & $T_{\mathrm{t} 1}$ & -50 to $+50^{\circ} \mathrm{C}$ & $1.5 \mathrm{mK}$ & $100 \mathrm{~Hz}$ & $0.15 \mathrm{~K}$ \\
\hline Total air temperature 2 & $T_{\mathrm{t} 2}$ & $-80^{\circ}$ to $+50^{\circ} \mathrm{C}$ & $2 \mathrm{mK}$ & $100 \mathrm{~Hz}$ & $0.15 \mathrm{~K}$ \\
\hline \multicolumn{6}{|c|}{ Humidity } \\
\hline Ly- $\alpha$ humidity absolute humidity & $a$ & $0.1-20 \mathrm{~g} \mathrm{~m}^{-3}$ & $10^{-4}-10^{-2} \mathrm{~g} \mathrm{~m}^{-3}$ & $100 \mathrm{~Hz}$ & $2 \%\left(4 \%\right.$ below $\left.0.5 \mathrm{~g} \mathrm{~m}^{-3}\right)$ \\
\hline TP3 dewpoint temperature & $T_{\mathrm{d}}$ & -30 to $+50^{\circ} \mathrm{C}$ & $0.015 \mathrm{~K}$ & $10 \mathrm{~Hz}$ & $0.35 \mathrm{~K}$ \\
\hline Humicap relative humidity & $h_{\mathrm{RH}}$ & $0-100 \%$ & $3 \times 10^{-3} \%$ & $10 \mathrm{~Hz}$ & $3 \% \mathrm{RH}$ \\
\hline
\end{tabular}

vice (e.g. $\pm 0.1 \mathrm{~K}$ ) and the TAT housing (e.g. $\pm 0.1 \mathrm{~K}$ ) also have to be considered, leading to an overall uncertainty of $\pm 0.15 \mathrm{~K}$. Within the TAT housing the airflow is almost reduced to stagnation. The correction of the so-measured total air temperature to the static temperature (the temperature of the undisturbed air) causes the above-mentioned error of the TAT housing. Due to the low indicated airspeed (IAS $<80 \mathrm{~m} \mathrm{~s}^{-1}$ ) of the Caravan, the temperature rise at the TAT housing does not exceed $3 \mathrm{~K}$ and, thus, the so-called "recovery correction" and also the expected errors are comparably small (Bange et al., 2013).

The humidity has a weak influence on the air density and hence also on the tas and the calculated wind speed. For the calibration of the three humidity devices, we use a universal humidity calibration unit with a mixed gas-flow humidity generator (HygroStar) (Mayerbuch, 2006). The mobile system is attached directly to the humidity channel at the aircraft, which allows one to include the entire measurement equipment in the calibration. In the closed system a wide range of pressure and humidity values are simulated and compared to a reference dewpoint mirror (MBW Calibration Ltd., model DPM 373 LX) which has an overall measurement uncertainty of $0.1 \mathrm{~K}$. For the Ly- $\alpha$ an additional inflight calibration method is applied to account for the change in radiation intensity during operation. The estimated measurement uncertainty for the Ly- $\alpha$ is listed in Table 3, where information about all relevant quantities for the wind calculation is summarised.

The contributors for the 3-D wind calculation, temperature and humidity are recorded with at least $100 \mathrm{~Hz}$, which corresponds to a horizontal resolution of $0.6 \mathrm{~m}$ during normal measurement flight conditions. The high resolution (see Table 3) of the data allows for turbulence measurements with the Caravan. A major contribution to the quality of this system is made by the IGI. It provides the position and attitude information for the aircraft with very high accuracy and $128 \mathrm{~Hz}$. As a consequence the remaining challenge is to optimise the calibration of the gust probe, which has the biggest contribution to the residing errors of the $3-\mathrm{D}$ wind estimation on a research aircraft.

\section{In-flight calibration}

The measurement of the tas on an airborne system is often realised with a 5HP mounted on a long boom to reach a point of minimal flow distortion ahead of the aircraft structure. Two main steps are necessary to quantify the residual errors contaminating the measurement of the static and dynamic pressures as well as the flow angles $(\alpha$ and $\beta$ ). The first part is a "static calibration" where correction functions for the quantities are derived during stabilised flight condi- 
tions. With the second step - the dynamic calibration - dependencies during changing flight conditions are quantified and corrected if the errors are significant. This step is also used to determine possible time offsets between the different quantities. When the 3-D wind data are calculated with a frequency of $100 \mathrm{~Hz}$, even minor time offsets will have a significant impact.

\subsection{Static pressure calibration}

The calibration process starts with the determination of the static source error $\left(\Delta p_{\mathrm{s}}\right)$ - the difference between the indicated static pressure $\left(p_{\mathrm{si}}\right)$ at the gust probe and the true static pressure $\left(p_{\mathrm{s}}\right)$ of the undisturbed air. For any aircraft and any specific mounting point of the gust probe, the magnitude of $\Delta p_{\mathrm{s}}$ is different. Furthermore, the flow field around the aircraft changes with speed and height, which is why $\Delta p_{\mathrm{s}}$ must be determined for the whole flight envelope (e.g. height and Mach number range) of the research aircraft. At the tip of the gust probe the total pressure $\left(p_{\mathrm{t}}\right)$ is measured, which is the sum of the static and the dynamic pressure $\left(q_{\mathrm{c}}\right)$. As a basic assumption $p_{\mathrm{t}}$ is conserved around the aircraft (within the angular acceptance range of the probe) and according to

$p_{\mathrm{s}}=p_{\mathrm{si}}-\Delta p_{\mathrm{s}} \quad$ and $\quad q_{\mathrm{c}}=p_{\mathrm{t}}-p_{\mathrm{s}}$

the correct dynamic pressure can also be estimated. Equation (2) shows the significance of $q_{\mathrm{c}}$ for the calculation of the TAS. Thus, the exact determination of $\Delta p_{\mathrm{s}}$ plays an important role in the wind calculation. A simple method for this is to compare $p_{\mathrm{si}}$ with a reference value ( $\left.p_{\text {ref }}\right)$ at the height of the pressure transducer in the aircraft. Gracey (1979) describes a method to calculate $p_{\text {ref }}$ from a reference value measured on the ground. The ground value $\left(p_{0}\right)$ is corrected for the height difference $\Delta h$ with the barometric height formula

$p_{\text {ref }}=p_{0} \cdot e^{-\frac{g \cdot \Delta h}{R \cdot \bar{T}_{v}}}$

The gravity $(g)$ and the vertical mean of the virtual temperature $\left(\bar{T}_{\mathrm{v}}\right)$ have a minor impact on the measurement error of the reference pressure, but already a small error in the height estimation is resulting in a significant pressure error (e.g. at sea level, $0.8 \mathrm{~m}$ leads to $\sim 10 \mathrm{~Pa}$ pressure error). While the height was usually determined from photographs or radar tracking in the past, the DPGS information from the IGI system can now be used, which leads to a significant reduction of the calibration error. Three major improvements for an optimised tower-flyby method are described in Mallaun and Giez (2013): (i) the IGI offers an increased accuracy of the height determination; (ii) the high-frequency data of the IGI can be used to correct for small height fluctuations and attitude changes during the stable measurement sequences; (iii) before and after the flight a "ground block"
Table 4. List of the calibration flights performed for the static and dynamic calibrations of the Caravan measurement system. Four tower-flyby (TFB) and four racetrack (RTR) flights were conducted to parametrise the static pressure error (dps), the attack angle $(\alpha)$, the sideslip angle $(\beta)$ and the dynamic pressure error $\left(\mathrm{dps}_{\mathrm{ab}}\right)$. The TFB flights were conducted near ground (e.g. $\sim 30 \mathrm{~m}$ above the runway), the RTR in flight levels FL100 and FL140 (e.g. $~ 3000$ and $\sim 4300 \mathrm{~m}$ a.s.1.).

\begin{tabular}{lrccccc}
\hline Flight & Date & dps & $\alpha$ & $\beta$ & $\mathrm{dps}_{\mathrm{ab}}$ & Level \\
\hline TFB no. 1 & 15 March 2011 & $\mathrm{x}$ & $\mathrm{x}$ & & & \\
TFB no. 2 & 21 March 2011 & $\mathrm{x}$ & $\mathrm{x}$ & & & \\
TFB no. 3 & 22 March 2011 & $\mathrm{x}$ & $\mathrm{x}$ & & & \\
TFB no. 4 & 23 March 2011 & $\mathrm{x}$ & $\mathrm{x}$ & & & \\
RTR no. 1 & 29 March 2011 & $\mathrm{x}$ & $\mathrm{x}$ & $\mathrm{x}$ & $\mathrm{x}$ & FL100 \\
RTR no. 2 & 22 August 2011 & $\mathrm{x}$ & $\mathrm{x}$ & $\mathrm{x}$ & $\mathrm{x}$ & FL100 \\
RTR no. 3 & 31 August 2011 & $\mathrm{x}$ & $\mathrm{x}$ & $\mathrm{x}$ & $\mathrm{x}$ & FL140 \\
RTR no. 4 & 28 March 2012 & & & $\mathrm{x}$ & $\mathrm{x}$ & FL100 \\
\hline
\end{tabular}

(short reference measurement with the aircraft on the runway) is performed and, thus, the pressure and height determinations become differential measurements reducing the measurement uncertainty. For the calibration of the Caravan system, the whole envelope of the slow aircraft could be tested during the four tower-flyby flights listed in Table 4, but to check on possible height dependencies of $\Delta p_{\mathrm{s}}$ three additional racetrack flights were performed with test patterns in flight levels (FL)100 and FL140 ( 3000 and 4300 m.s.l., respectively). The racetrack pattern is a rectangular flight sequence with predefined measurement legs which is repeatedly flown through during the calibration flight. The simplest possible calibration scheme - calculating $\Delta p_{\mathrm{s}}$ with a thirdorder polynomial as a function of dynamic pressure - gave the best results where any height dependency vanished. The common approach to parametrise with Mach number and the height turned out to be less successful, which might be due to the limited speed and height range of the Caravan. The parametrisation distinguishes between clean configuration and a $10^{\circ}$ setting of the flaps (flaps10), which has a significant impact on the flow around the aircraft. The flaps 10 configuration is advantageous when the aircraft flies with low speeds and small attack angles, as is often desired during measurement flights. The results of the calibration are shown in Fig. 4 (reproduction of Fig. 10 in Mallaun and Giez, 2013) depicting the difference between the corrected pressure of the gust probe and the reference value for a total of 96 test points with clean and flaps 10 configurations covering the whole speed envelope of the aircraft. The black error bar represents the estimated overall accuracy of the static pressure measurement of $\pm 25 \mathrm{~Pa}$. From these results a standard deviation (SD) $(\sigma)$ of $\pm 10 \mathrm{~Pa}$ for the error of the static pressure calibration can be calculated, and the overall measurement uncertainties for the static pressure and the dynamic pressure sum up to \pm 25 and $\pm 15 \mathrm{~Pa}$, respectively. 


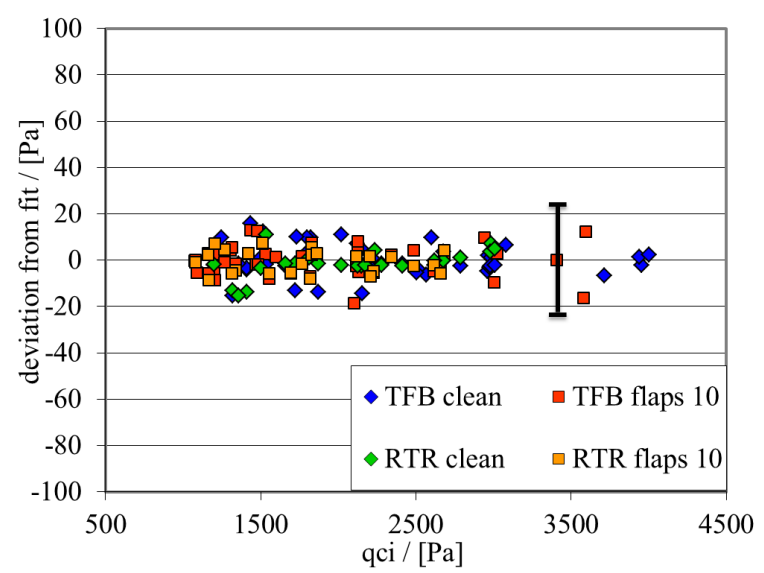

Figure 4. Results of the pitot-static calibration: deviation of the static source error $\left(\Delta p_{\mathrm{S}}\right)$ of the third-order polynomial fit for all tower-flyby (TFB) and racetrack (RTR) flight points. The black error bar represents the estimated overall accuracy of the static pressure measurement $(\sigma \sim 0.25 \mathrm{hPa})$.

\subsection{Static flow angle calibration}

The performance of the 5HP has been thoroughly tested and characterised by the manufacturer in the laboratory and in the wind tunnel (De Leo and Hagen, 1976). According to this the indicated flow angles $\left(\alpha_{i}\right.$ and $\beta_{i}$ in $\left.^{\circ}\right)$ are calculated with

$\alpha_{i}=K^{-1} \cdot \frac{\mathrm{d} p_{\mathrm{a}}}{q_{\mathrm{c}}} \quad$ and $\quad \beta_{i}=K^{-1} \cdot \frac{\mathrm{d} p_{\mathrm{b}}}{q_{\mathrm{c}}}$,

$K=0.0789+0.0001 \cdot M a$ and $M a<0.5$

using the fraction of the differential pressure of the respective axis $\left(\mathrm{d} p_{\mathrm{a}}\right.$ or $\left.\mathrm{d} p_{\mathrm{b}}\right)$ over the dynamic pressure $\left(q_{\mathrm{c}}\right)$ multiplied by a coefficient $K^{-1}$. This linear relation is valid for flow angles $\alpha ; \beta<10^{\circ}$ and the $K$ value has a weak Mach number (Ma) dependency. With these definitions the indicated flow angles can be calculated at the position of the gust probe, but due to flow distortion around the aircraft these values will not represent the undisturbed flow. Most of the difference is caused by upward deflected air masses in front of the wing, the "upwash", which is generated through the lift of the aircraft. This leads to a significant overestimation of the attack angle. Boegel and Baumann (1991) describe a method to calculate modified $K$ values that account for this flow modification, while Kalogiros and Wang (2002) use a simplified model of the aircraft dynamics to calculate and correct the upwash component on the vertical wind. We propose a method where first the indicated flow angles are calculated with Eq. (13) (as these angles are true for the actual conditions at the 5HP position) and correct the dynamic effects with a parametrisation derived from well-designed test flight programs.

\subsubsection{Attack angle calibration}

Under stable horizontal flight conditions the attack angle $(\alpha)$ is equal to the pitch angle $(\Theta)$ (Haering Jr., 1995). Flying test points for the whole speed envelope of the aircraft, a wide range of different $\alpha$ values can be realised. The test points performed for the static pressure calibration during seven test flights, as listed in Table 4, are perfectly suitable for this calibration step. The biggest adverse impact on the calibration comes from vertical velocities of the aircraft and vertical winds, but the influence of attitude changes of the aircraft also has to be considered. Therefore, optimum weather conditions are needed for the test flights to perform stabilised test points for the calibration. Minimum turbulence can be found in the early morning during the cold season. Weak high-pressure influence guarantees slow changes in the environmental properties (e.g. pressure, temperature and wind), low wind speeds and small horizontal pressure gradients. Aligning the test patterns along the geostrophic wind direction reduces the effects of the horizontal pressure gradient, and regions of very stable stratification should be omitted, because of strong vertical gradients of the temperature and wind fields. For each of the 96 test points, an interval is chosen manually where the aircraft is in stabilised straight and level flight (e.g. no change in altitude, attitude angles or speed). For these intervals average values for the indicated alpha $\left(\alpha_{\text {ind }}\right)$ and the pitch angles are calculated. The comparison of the $\alpha_{\text {ind }}$ values with the reference values for all the successful test points is shown in Fig. 5a. A linear relation of the flow deflection is found and again a difference between clean (blue diamonds) and flaps10 (red diamonds) configurations is visible. The difference between the data points and the grey $1: 1$ line in the plot at $\alpha_{\text {ind }}=0$ represents the vertical offset angle $\left(\varepsilon_{\mathbf{b}}\right)$ between the nose boom and the IGI system. For both configurations this offset is about $2.5^{\circ}$, which implies that the $4^{\circ}$ tilt of the nose boom is significantly compensated for by the upwash. Its impact is strongly dependent on the airspeed and thus also on $\alpha_{\text {ind }}$. For the correction of the attack angle, a linear approach is sufficient. The offset angle $\varepsilon_{\mathrm{b}}$ is calculated for each individual flight with the method demonstrated in Sect. 2.2, because this offset might change after a new system integration. The results of Fig. 5a are used to estimate the linear coefficient, which is $\sim 0.78$ and $\sim 0.77$ for the clean and flaps 10 configurations, respectively (i.e. $\alpha_{\mathrm{NB}, \text { clean }}=0.78 \cdot \alpha_{i}$ ). The deviations of the corrected $\alpha$ values from the reference values are shown in Fig. 5b. This parametrisation corrects all the flow-induced effects around the aircraft on the attack angle during stable flight conditions as well as the uncertainty of the 5HP calibration (e.g. the $K$ value). To calculate the measurement uncertainty of the $\alpha$ calibration, we performed a cross-validation (e.g. Wilks, 2006) where five random samples of the clean and an additional five of the flaps 10 configuration were omitted in each run and used as independent test samples. The result of this test is a $\mathrm{SD} \sigma=0.2^{\circ}$ for the attack angle calibration. 

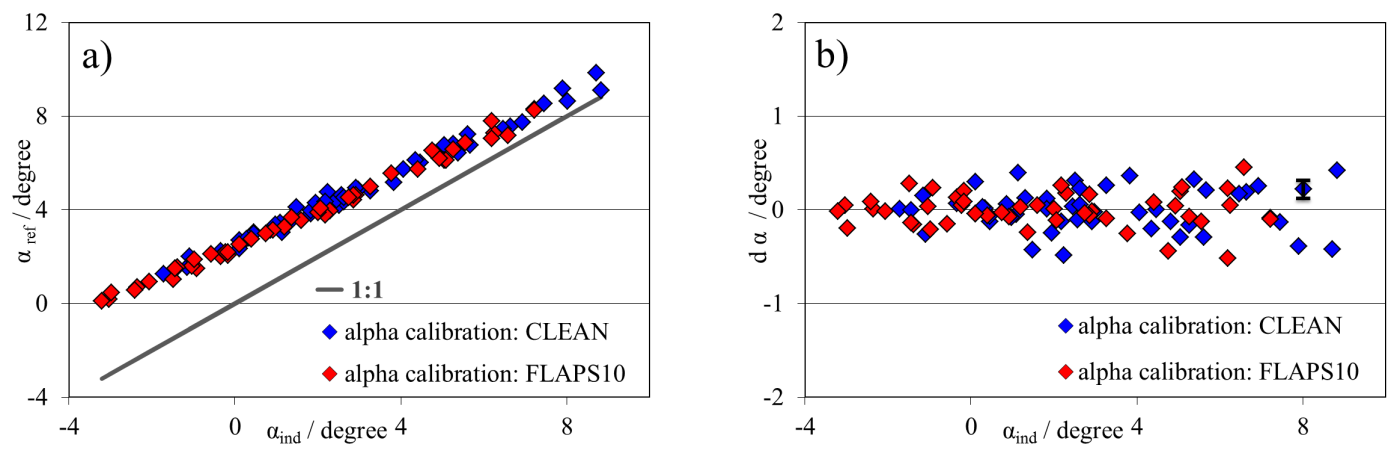

Figure 5. (a) Comparison of $\alpha_{\text {ind }}$ with the measured reference values $\alpha_{\text {ref }}=\Theta$ for 96 test points during six calibration flights. (b) Deviation of the corrected $\alpha$ values and the reference values $(\Theta)$. A linear fit is performed with the results shown in (a) to correct the upwash effect for clean and flaps10 configurations.

\subsubsection{Influence of the flow angles on the pressure}

The expected next step would be the calibration of the sideslip angle $\beta$, but before this is possible, we have to account for the influence of the flow angles on the pressure measurement. This is necessary because changes in $\alpha$ and $\beta$ modify the flow field around the aircraft and, thus, also the static pressure error (Boegel and Baumann, 1991). Equation (2) shows the importance of a correct dynamic pressure for the TAS calculation, which again is needed for the $\beta$ calibration. To parametrise the pressure error induced by the sideslip angle $\left(\mathrm{dps}_{\beta}\right)$, we have to use the indicated sideslip $\left(\beta_{\mathrm{i}}\right)$, as the corrected $\beta$ is not yet available. However, this is the correct choice anyway, because the orientation of the gust probe is more stable compared to the IRS and gives the better reference. This is due to the fact that the mounting points of the METPOD lead to a fixed position of the gust probe, while the orientation of the reference platform might slightly change from one system integration to the other within the tolerance of the mounting studs on the seat rails in the cabin. The steady sideslip manoeuvre is a simple method to estimate $\mathrm{dps}_{\beta}$, where different constant sideslip angles are induced during stable horizontal flight conditions. Crossing aileron and rudder the pilot forces a constant sideslip with different deflections, while a small roll angle is needed for a constant flight direction. The sideslip angle is kept stable for several seconds before the next test point is performed with a different $\beta$. For good test points favourable weather conditions are needed as described in Sect. 4.2.1 and some experience for the test pilots is indispensable. It is possible to realise deflections equal to situations of strong turbulence where the maximum angles of $\beta$ occur (e.g $\sim \pm 10^{\circ}$ ). Similar to the discussion in Sect. 4.1 the pressure signal must be corrected for height changes during the manoeuvre using Eq. (12), the barometric height formula. Again, the high accuracy of the DGPS height measurement is an important factor for a successful calibration. An example of two sequences of the steady sideslip manoeuvre is given in Fig. 6a. The upper panel shows the time series of $\beta_{\mathrm{i}}$ where deflections of up to $\pm 7^{\circ}$ were induced. In the lower panel the black line represents the original pressure signal and the red line is the height-corrected pressure, where finally the sideslip dependency of the pressure signal becomes visible. The manoeuvre starts with several seconds of stable straight level flight, followed by several seconds of steady sideslip with different deflections to both sides. In the middle and at the end of the manoeuvre the straight level flight is performed again. This sequence lasts about 3-5 min and is repeated in both flight directions with clean and flaps 10 configurations to collect reliable calibration data. The pressure reference value for the height correction in Eq. (12) is taken at the beginning of the time series during a steady straight level flight. For a valid test point the mean of at least $4 \mathrm{~s}$ of constant $\beta_{\mathrm{i}}$ was taken. Corresponding to the information in Table 4, the manoeuvre was repeated during four test flights. For the parametrisation we evaluated 159 successful test points which are shown in Fig. 6b. During stable straight level flight the expected $\operatorname{dps}_{\beta}=0$ is found for slightly positive sideslip angles. While here the deviation of the pressure signal is well within the measurement uncertainty calculated in Sect. 4.1, it reaches values of up to $-150 \mathrm{~Pa}$ for big positive sideslip angles. The asymmetric behaviour of the deviation comes from the position of the gust probe under the left wing. The scatter of the results is slightly enhanced for positive angles compared to the negative ones, which can also be an effect of the asymmetry. To correct the pressure signal, a second-order polynomial fit is calculated from all the test points. There is no significant dependency on the aircraft configuration visible.

A similar calibration procedure can be realised for the $\alpha_{\mathrm{i}}$ dependency of the pressure $\left(\mathrm{dps}_{\alpha}\right)$. The height-corrected pressure signal is correlated with $\alpha_{\mathrm{i}}$ during the pitch oscillation manoeuvre which is described in detail in Sect. 5. The pilot induces a periodic vertical oscillation of the aircraft with a period of several seconds. While the attack angle is oscillating as well, the speed of the aircraft is kept nearly constant. For the current installation on the Caravan we found values of $\mathrm{dps}_{\alpha}$ that are clearly smaller than the measurement 

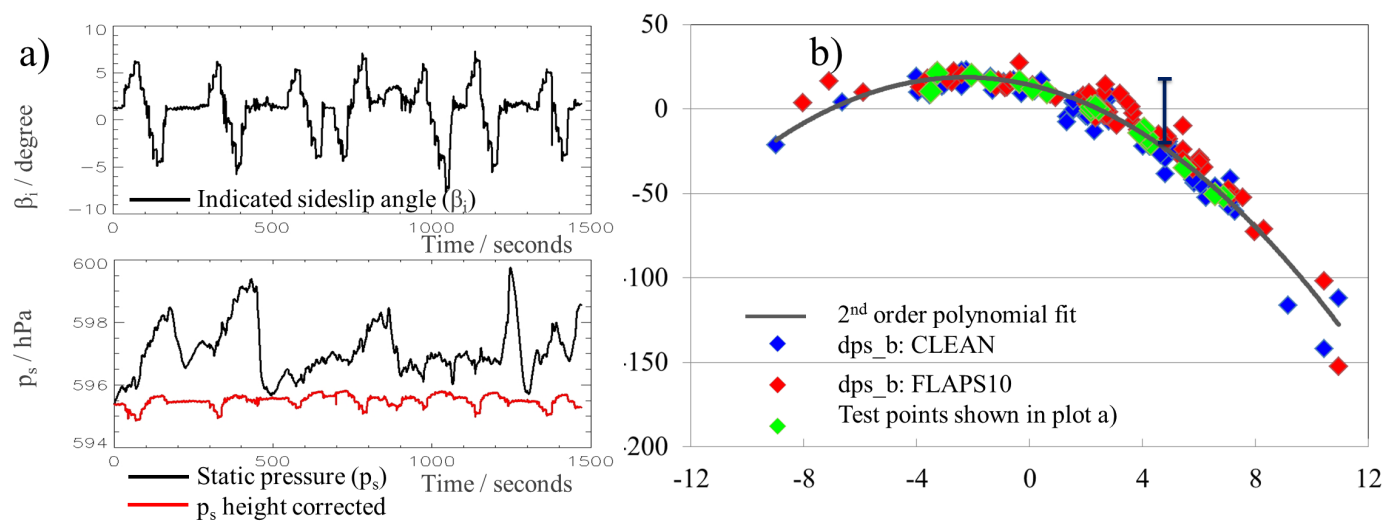

Figure 6. (a) Example of two sequences of a steady sideslip manoeuvre lasting about $500 \mathrm{~s}$. The upper panel shows the indicated sideslip angle and the lower panel the corresponding static pressure signal. The raw pressure signal is dominated by the height variation of the aircraft and the $\beta$ dependency becomes visible when the signal is height corrected using Eq. (12). (b) Difference between the static pressure signal and the reference value calculated with Eq. (12) for 159 test points. Each test point is an average of at least $4 \mathrm{~s}$ of a constant steady sideslip angle. A second-order polynomial fit is used to correct the static pressure signal from this $\beta$ influence. The green diamonds correspond to the test points shown in (a) and the black error bar represents the estimated accuracy of the pressure sensor $(\sigma \sim 20 \mathrm{~Pa})$.

uncertainty of the pressure sensor. Therefore, there is no need to implement it in the static pressure correction procedure. Different to $\beta$, the value of $\alpha$ is strongly correlated with the airspeed. The static pressure calibration in Sect. 4.1 is based on the airspeed (through $q_{\mathrm{c}}$ ) and already includes the major parts of the $\alpha$ dependency.

\subsubsection{Sideslip angle calibration}

The $\beta$ calibration is more complicated compared to $\alpha$, because there is no simple equivalent to obtain the reference value for the sideslip angle $\left(\beta_{\text {ref }}\right)$. Khelif et al. (1999) suggest an iterative method minimising the errors in the wind calculation, while Haering Jr. (1990) presents an algorithm where the coefficients are derived from a reconstruction of the flight trajectory including Kalman filtering. Both methods need some a priori knowledge of the desired results and it is not possible to directly calibrate the sideslip angle or distinguish the influence of the different constituents involved in the analysis. We suggest that again the steady sideslip manoeuvres are a possibility to overcome these deficiencies as they provide a possibility to calibrate $\beta$ during static flight conditions. From the wind equation (Eq. 1), $\beta_{\text {ref }}$ is calculated and then used to parametrise the $\beta$ correction. The second component (index $y$ ) of Eq. (1)

$\boldsymbol{t a} \boldsymbol{s}_{y f}=g s_{y f}-v_{f}+L_{y f}$

is taken in the aircraft fixed coordinate system (index $f$ ). $v_{f}$ is the cross-wind component rectangular to the aircraft longitudinal axis and $L_{y f}=(\boldsymbol{\Omega} \times \boldsymbol{L})_{y f}$ represents the correspondent rotational component of the lever arm. The exact calculation of the second component of the true airspeed in Eq. (3) is simplified to

$\operatorname{tas}_{y f}=\mathrm{TAS} \cdot \sin \left(\beta_{\mathrm{ref}}\right)$ without introducing significant errors for typical angles of attack (Lenschow and Spyers-Duran, 1989). Inserting Eq. (16) in Eq. (15) leads to

$\beta_{\text {ref }}=\arcsin \left(\frac{g s_{y f}-v_{f}+L_{y f}}{\text { TAS }}\right)$,

a simple formulation to directly calculate $\beta_{\text {ref }}$ from the wind equation. A visualisation of the method is shown in Fig. 1b where the different components in Eq. (17) are represented by the dotted lines (for clarity $L_{y f}$ is not included). For the $\beta$ calibration the steady sideslip manoeuvre is performed as described in Sect. 4.2.2 and shown in Fig. 6a. The same valid test points are used also for this calibration step. With Eq. (17) we calculate $\beta_{\text {ref }}$ for the 159 test points already described in the last subsection. Again, just the mean values of the involved parameters are used for the calculation with averaging periods of at least $4 \mathrm{~s}$ of constant sideslip. Even though $\beta$ is not yet finally corrected, a correct mean wind signal can be obtained. We apply the method described in Sect. 2.2 to obtain the correction coefficients for the different orientations of the gust probe and the IRS in order to calculate a reliable wind signal. During the calibration sequences with induced $\beta$ the wind will be corrupt. Therefore, we built a running average of $5 \mathrm{~min}$ on the wind data, which corresponds to the approximated duration of one test series. Alternatively the wind data during straight level flight could be taken for the wind correction, which did not result in any visible improvement. The importance of calm conditions with little turbulence becomes obvious during this discussion. Any wind changes between the single test sequences will perturb and increase the scatter of the results.

The correlation of $\beta_{i}$ and the calculated $\beta_{\text {ref }}$ for the 159 test points is shown in Fig. 7a, where the linear relation becomes clearly visible. The difference between the measured 

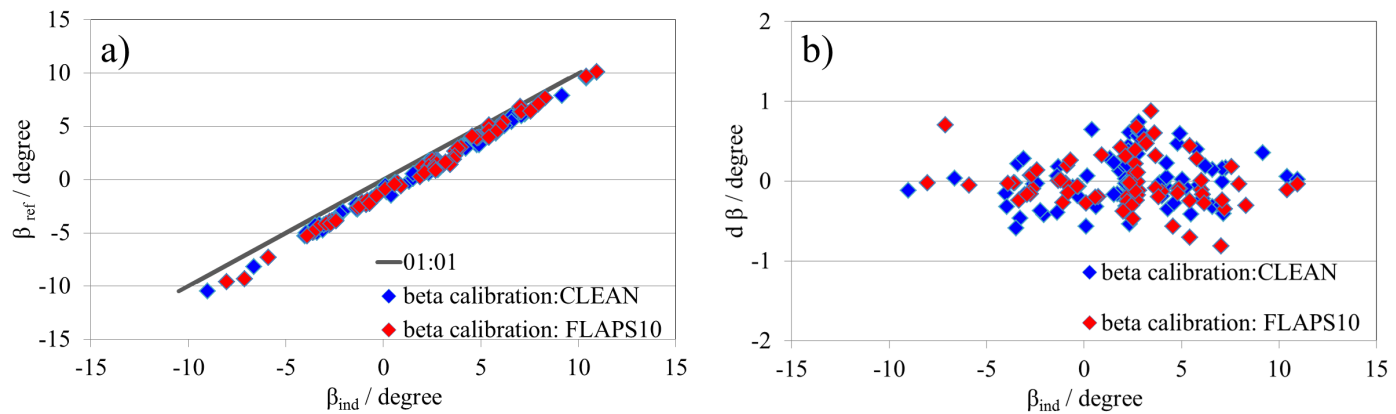

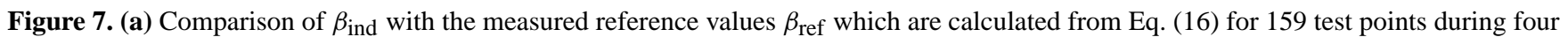
calibration flights. (b) Difference between the corrected $\beta$ values and the reference values ( $\beta_{\text {ref }}$ ). A linear fit is performed with the results shown in (a) to correct the sidewash effect for all the test points. The scatter of the results is due to short-time changes of the wind speed during the calibration manoeuvres.

values and the indicated values (i.e. the sidewash effect) is much smaller compared to the upwash effect for $\alpha$. The calibration shows an underestimation of $\beta$ of just $4 \%$ (i.e. $\beta_{\mathrm{NB}}=1.04 \cdot \beta_{i}$ ). A linear fit is applied to correct the indicated sideslip data, with the results shown in Fig. 7b. While the necessary correction of the flow angle is very small, the scatter of the data $\left(\sigma \sim 0.3^{\circ}\right)$ is rather high due to the wind variability. Therefore the scatter is too large for an estimation of the true calibration uncertainty of $\beta$. More realistic is the separation of a mean offset error and a relative error for $\beta$. The latter corresponds to the uncertainty of the linear coefficient for the $\beta$ parametrisation. The variability of the linear calibration coefficients calculated separately for the four test flights is $1 \%$, which will be taken as the relative error (i.e. $\left.\beta=(1.04 \pm 0.01) \cdot \beta_{i}+\eta_{\mathrm{b}}\right)$. We define the mean offset error of $\beta$ as the variability of the coefficient $\eta_{\mathrm{b}}$ (compare Sect. 4.3) which includes all the mean offset errors of the sideslip angle relative to the reference CS defined by the IRS. It includes any misalignment of the gust probe and the IRS as well as the errors from dynamical effects of the aircraft (e.g. the sidewash effect).

\subsection{Calculation of the flow angle offsets $\varepsilon_{b}$ and $\eta_{b}$}

For the wind calculation of the Caravan system, the flow angle offsets $\varepsilon_{\mathrm{b}}$ and $\eta_{\mathrm{b}}$ are calculated with the method described in Sect. 2.2. The results for the 43 Caravan flights since 2011 are visualised in Fig. 8, where each data point represents the correction value for one specific flight. Although the results of the Dassault Falcon20 in Fig. 2 are based on a larger sample, the main features of the $\eta_{\mathrm{b}}$ and $\varepsilon_{\mathrm{b}}$ calculation remain the same also for the Caravan. While at the beginning and the end of the series of $\eta_{\mathrm{b}}$ in Fig. 8 the scatter is relatively small, there is more noise visible for flights 13-30. Some of these flights were too short, but technical and calibration difficulties also had to be solved before the results improved again. The significant step between flights 35 and 36 is caused by the reintegration of the system into the aircraft. A slightly different orientation of the IRS of about $1^{\circ}$ was causing this

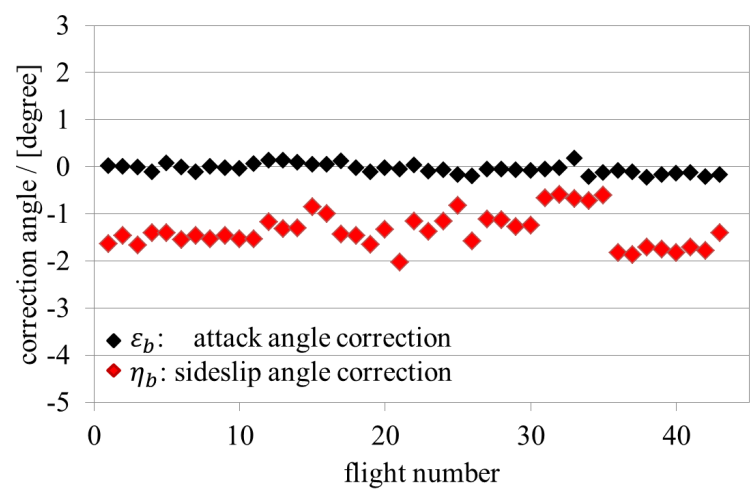

Figure 8. Results of the flow angle correction for 43 flights with the Caravan system after 2011. The correction for the attack angle offset shows significantly less scatter than for the sideslip angle. While the measurement system on the Falcon20 (see Fig. 2) is permanently installed, the system in the Caravan has to be reintegrated for each flight campaign. This leads to steps in the magnitudes of $\eta_{\mathrm{b}}$ after a new integration of the measurement equipment.

effect. In the vertical axis this effect is not visible. For the quality of the flow angle determination with respect to the IRS the calculation of $\varepsilon_{\mathrm{b}}$ and $\eta_{\mathrm{b}}$ is crucial. The variability of the two correction angles allows for an estimation of the measurement uncertainty, which is $\sigma=0.1^{\circ}$ for $\alpha$ and $\sigma=0.2^{\circ}$ for $\beta$. The offsets due to new system integrations should not contribute to the error of $\beta$. Therefore, $\sigma$ is at first calculated separately for each integration period and later averaged. Finally, to gain the overall error of the flow angles, we have to build the sum of the three error sources: (i) the relative flow angle error described in Sect. 4, (ii) a minor contribution from the uncertainty of the pressure transducers, and (iii) the variability of the correction angles $\varepsilon_{\mathrm{b}}$ and $\eta_{\mathrm{b}}$. 

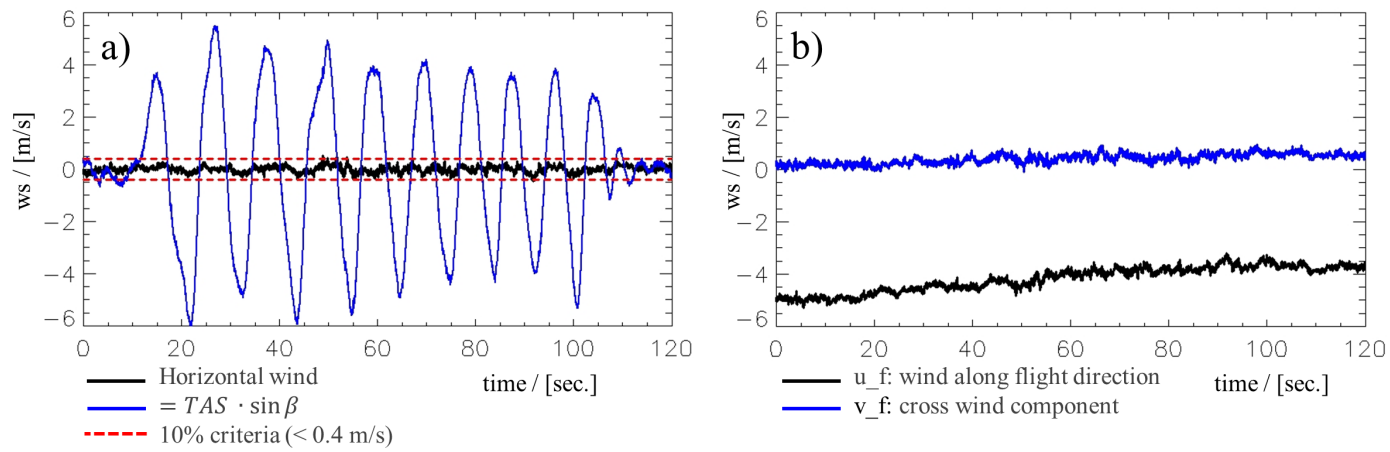

Figure 9. Results of the horizontal wind calculation during harmonic yaw oscillation. (a) shows the comparison of the horizontal wind with the induced cross-wind component. In order to point out the residual error due to the induced oscillation, the mean wind is removed. The criterion for an accurate calibration is that the residual error of the horizontal wind be less than $10 \%$ of the induced cross-wind component (e.g. TAS $\cdot \sin \beta_{\max } \sim 0.4 \mathrm{~m} \mathrm{~s}^{-1}$ ). In (b) the wind components along and perpendicular to the flight direction are drawn. The former is influenced by a possible error of the true airspeed and the latter of the sideslip angle.

\section{Dynamic pressure calibration}

Applying the steps in Sect. 4, the calibration of all the involved parameters for the wind calculation is completed. At this point all parameters are properly calibrated. However, possible time shifts in the acquisition would cause problems in the calculation of physical parameters. Boegel and Baumann (1991) describe a method on how to correct for these time effects by introducing manoeuvres with harmonic oscillations in the vertical and horizontal directions during test flights. As the wind measurement has to be independent of the aircraft motion, these oscillations must not appear in the calculated wind data. The authors demonstrate how effectively these contributions can be reduced by shifting the time series according to estimated time delays of the individual signal sources. For the Caravan no significant time delays are expected for the attitude and position data from the IGI system. They should be small (i.e. $<0.1 \mathrm{~s}$ ) also for the pressure signals. Dampening in the pressure tubes might have a possible influence (Hauf, 1984).

\subsection{Harmonic yaw oscillation}

The harmonic yaw oscillation manoeuvre is performed during all four racetrack test flights listed in Table 4 . After a $30 \mathrm{~s}$ period of steady flight the pilot crosses rudder and aileron inducing a sideslip angle of several degrees deflection in one direction without inducing a significant roll angle. By inverting the rudder input repeatedly a harmonic yaw oscillation with a period of about $10 \mathrm{~s}$ is achieved. Pilot experience is needed to complete about 10 periodic cycles without significant roll angles, heading changes or height losses. The example in Fig. 9 shows a successful manoeuvre with 10 full oscillations with an amplitude of $4^{\circ}$ and a period of about $10 \mathrm{~s}$ flying almost opposite to the mean wind direction. The black line shows the horizontal wind fluctuations calculated with no time shifts. A running mean of the original data by
$20 \mathrm{~s}$ is subtracted to make any residual dependencies of the yaw oscillation visible. In Fig. 9a this signal is compared to an artificial cross-wind calculated from $v_{\mathrm{a}}=\mathrm{TAS} \cdot \sin \beta$ (compare Eq. 16). Lenschow and Spyers-Duran (1989) proposed this criteria as a quality measure for the dynamic wind calculation. A residual error of $10 \%$ in the wind signal is accepted according to this criteria. In the example the fluctuation should not exceed $\pm 0.4 \mathrm{~m} \mathrm{~s}^{-1}$ (marked with the red dots), which is well fulfilled. Furthermore, the major part of the residual fluctuation is not correlated with the yaw oscillation. In Fig. 9b the wind components along and perpendicular to the flight direction are plotted separately. While the former would be sensitive to errors in the TAS calculation, the latter would indicate any deficiencies of the $\beta$ calibration. The results prove that without any further calibration or time shifts the horizontal wind calculation shows no significant contribution of the aircraft movements. However, it is important to note that this test does not give any information on the quality of the mean horizontal wind.

\subsection{Harmonic pitch oscillation}

A similar test is performed for the vertical wind component. The pilot induces a harmonic pitch oscillation via pulling and pushing the elevator. This manoeuvre is easier to realise than the yaw oscillation, but as before it is important to keep direction and altitude constant. Figure 10 shows one example of the 25 repetitions of the manoeuvre during the four test flights with a period of $7 \mathrm{~s}$ and an amplitude of $3^{\circ}$ leading to a maximum vertical velocity of the aircraft of about $\pm 3 \mathrm{~m} \mathrm{~s}^{-1}$. The vertical wind is then compared to the vertical velocity of the aircraft and, similarly to the previous discussion for the yaw manoeuvres, the former should show less than $10 \%$ of the velocities of the latter. For the vertical wind, small deviations from zero can be detected, but no significant correlation with the vertical velocity of the aircraft is visible. Again, no time shifts or further correction to the measured 


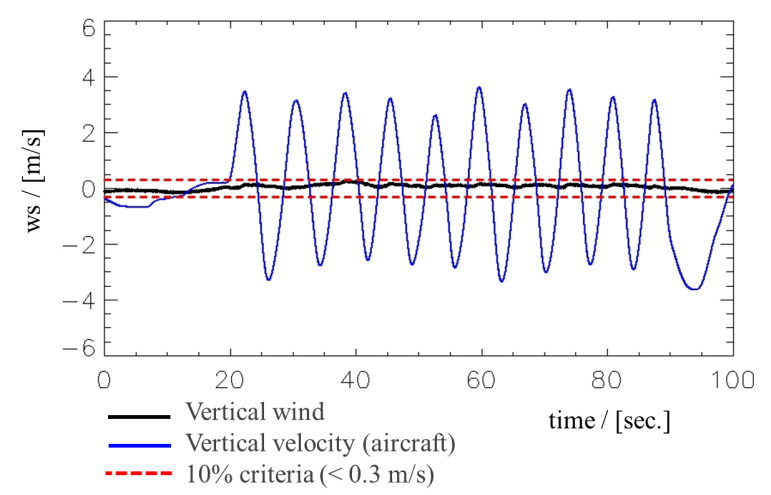

Figure 10. Results of the vertical wind calculation during harmonic pitch oscillation. The sinusoidal signal can clearly be seen in the vertical velocity of the aircraft, while it vanishes completely in the vertical wind component. The criterion for an accurate calibration is that the residual error of the vertical wind is less than $10 \%$ of the vertical velocity (e.g. $0.3 \mathrm{~m} \mathrm{~s}^{-1}$ ).

signals are necessary in order to optimise the wind calculation. It is a big advantage to avoid additional adjustments to the derived parameters in this stage of the calculation. The single components react very sensitively to any changes of coefficients or time shifts, while it is hardly possible to detect the true error source at the end of the wind calculation procedure. The small residuals of the vertical wind in Fig. 10 could be natural (e.g turbulence, terrain effects). The error calculation in Sect. 6 will show that this offset does not exceed the estimated measurement uncertainty of the vertical wind.

\subsection{High-frequency wind data}

For turbulence measurements the data acquisition system logs all relevant parameters with $100 \mathrm{~Hz}$, which leads to a horizontal resolution of $0.6-0.7 \mathrm{~m}$ for a typical flight situation. The accuracy of the position and attitude data is guaranteed up to this frequency by the manufacturer, but the quality of the high-frequency pressure signal needs to be reviewed. The response times of the transducers are fast enough, but in the $\sim 2 \mathrm{~m}$ long pressure tubes, resonance effects as well as diminution of the signal can occur. Furthermore, the vibration of the nose boom can have an adverse impact (e.g. Hauf, 1984; Lenschow and Spyers-Duran, 1989). Figure 11 shows a comparison of the power spectra of the three wind components where these difficulties and their impact on the data quality become visible. The data represent a 6 min long flight leg within the turbulent boundary layer at a height of $\sim 1800 \mathrm{~m}$ above the ground. For the data evaluation, the horizontal wind was rotated towards the mean flight direction, thus, the $u$ and $v$ component are along and perpendicular to the flight direction, respectively. The aircraft was flying along the mean westerly wind which had a magnitude of $\sim 6 \mathrm{~m} \mathrm{~s}^{-1}$. For the spectral analysis, the time series were detrended. The

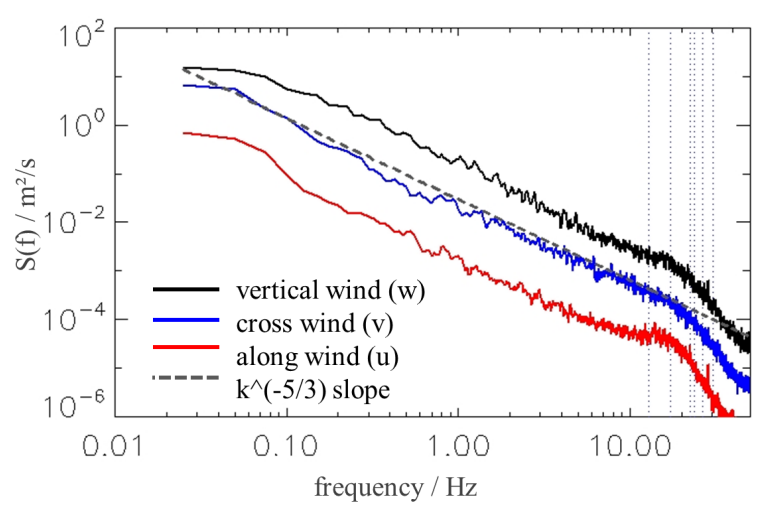

Figure 11. Power spectra of the three wind components in the aircraft coordinate system. The results of the vertical wind component $(w)$ and the along-wind component $(u)$ are shifted by 1 decade upwards and downwards relative to the cross wind $(v)$, respectively. The blue dotted lines on the right-hand side correspond to the resonance frequencies of the METPOD and the aircraft as measured during a ground vibration test. For the spectral analysis, the data were detrended and the spectra bin-averaged with $0.1 \mathrm{~Hz}$.

plot shows bin averages of $0.1 \mathrm{~Hz}$ where the $w$ component is shifted 1 order of magnitude upwards and the $u$ component downwards for better visibility. The $k^{-5 / 3}$ line in the vicinity of the cross-wind component represents the theoretically expected shape of the inertial subrange which is present in the data between 0.05 and $10 \mathrm{~Hz}$. An enhancement of the signals is visible between 15 and $20 \mathrm{~Hz}$, which is most significant for the along-wind component. Following the argumentation of Hauf (1984) this is due to resonance effects in the pressure tubes. Since the Caravan and the cited aircraft have similar tubes in terms of quality, diameter and length, a resonance frequency for the Caravan system of $15-20 \mathrm{~Hz}$ is very probable.

Above this frequency the attenuation of the fluctuation due to dampening effects within the tubes becomes visible. No white noise contribution can be detected, which would counteract this decay in the power spectrum. Also, the vibrations of the nose boom influence the power spectra, where the major impact is expected in the vertical and cross-wind directions due to the eigenfrequency of the $2 \mathrm{~m}$ long boom. A ground vibration test conducted in 2004 for certification purposes determined the strongest vibrations of the boom at $17.3 \mathrm{~Hz}$, but also at $12.8,22.5,23.8$ and $30.6 \mathrm{~Hz}$ an oscillation of the boom and the instrument container were detected. These frequencies are plotted with blue dashed lines in Fig. 11, but no corresponding signal in the power spectra (e.g. a sharp peak at the expected frequencies) of the different components can be observed. Thus, the vibration of the boom seems to have minor impact for the measured wind fluctuations. In some situations a significant peak appears in the spectra of the cross-wind component at frequencies between 29 and $31 \mathrm{~Hz}$, which seems to correspond to the rotation of the propeller (e.g. 1750-1900 rpm) and its pressure pertur- 
bance. It can be stated that up to $10 \mathrm{~Hz}$ the measurement system captures the wind fluctuations without any difficulties, but beyond this frequency the above mentioned effects have to be taken into account.

\section{Error analysis}

In the previous sections we derived the measurement uncertainties of the various parameters which are needed for the wind calculation. The respective results are summarised in Table 3 including all errors such as sensor errors, temperature dependencies, analogue conversion or aerodynamic effects. All these errors contribute to the overall uncertainty in the 3-D wind determination. It is of great interest to quantify this final error and to determine the individual contributions from the different input parameters. Druee and Heinemann (2013) propose the Gaussian error propagation and a differential error analysis as two possible ways for the respective error estimation. They describe limitations for both methods such as the difficult calculation as a consequence of the complexity of the system and the necessity to neglect minor terms. The complex and extensive algorithm used in the calculation of airborne wind measurements does not allow for an analytical solution in the error determination. Therefore, existing error analyses are usually based on the application of major simplifications and negligence to the existing processing schemes.

\subsection{Determination of error propagation}

In this paper we demonstrate a simple, effective and robust alternative to these methods, which yields a precise result even for very complex data processing schemes (Giez et al., 2005). The method implements true error propagation through the data calculation. The basic idea of this method is that the overall error (e.g. biases, random error or other dependencies) of a single measurement parameter is added to the original data time series as a white noise signal with the amplitude of the error. This white noise signal is added to all the different original time series, each with its individual measurement uncertainty. In other words: the uncertainty of a measurement parameter is expressed by adding artificial white noise to the original data just before the actual processing starts. When the calculation of derived quantities (e.g. 3-D wind, temperature or humidity) is performed with this modified data set, the added errors will propagate and superpose on the result. The cumulative amount of white noise in the processed data is then a measure for the propagated error sources from all input parameters. The deviation found in the results represents the exact impact of the applied errors (noise amplitude) and represents the complete error propagation even in complex and nonlinear systems. For this discussion it is completely irrelevant whether the applied offset represents a systematic bias or a random error. The method simply shows the propagation of any kind of error bandwidth into the processed data. An additional bias in the sensor data is nothing else than a new source of uncertainty with a given bandwidth ("error") around the mean value which must be included in the overall error. Therefore, random error and bias effects can be treated in one single step by using a combined error amplitude represented by the white noise.

The method benefits from the basic properties of a white noise signal: (i) the mean value of the added signal is zero; that is, the mean value of the input parameters is left unchanged. (ii) The white noise time series is represented by a Gaussian data distribution which is in accordance with the classical error model. (iii) White noise data points are statistically independent of each other, which allows for an easy identification of the white noise contribution to a time series by means of autocovariance.

The calculation of the error propagation with this method is realised by the following four steps.

i. The original data set is processed with the existing algorithms. The calculated results are analysed with respect to their "natural" white noise contributions caused by the sensors themselves or the data acquisition. It is interesting to note that for the measurement system discussed in the current paper no white noise is visible in the raw data.

ii. An artificial white noise signal is added to all the original data time series (in our case: position and attitude, other avionic data such as ADC pressure or ADC temperature and all pressure, flow angles, temperature or humidity signals of the METPOD). The amplitude of this signal represents the size of the error for the respective data, while a specific initial value of random numbers (i.e. a software-specific "seed value" in a pseudorandom number algorithm) for each parameter ensures that no correlation occurs between the different white noise signals. However, in some cases a correlation between different parameters does actually exist. One example is the uncertainty in the static source correction of the static and dynamic pressure. In this case one can use identical seed values but different signs for these input parameters.

iii. The modified data are processed again using the same algorithms as before.

iv. The calculated data are then analysed for their white noise contribution by means of autocovariance. This analysis is done for a short time interval with a sufficient number of data points for statistical reasons. A comparison of these results with the original data set directly yields the error contribution to the specific input parameters.

Figure 12 demonstrates the principles of this method and the use of the autocovariance function $\Psi_{x x}(\tau)$ which is used 

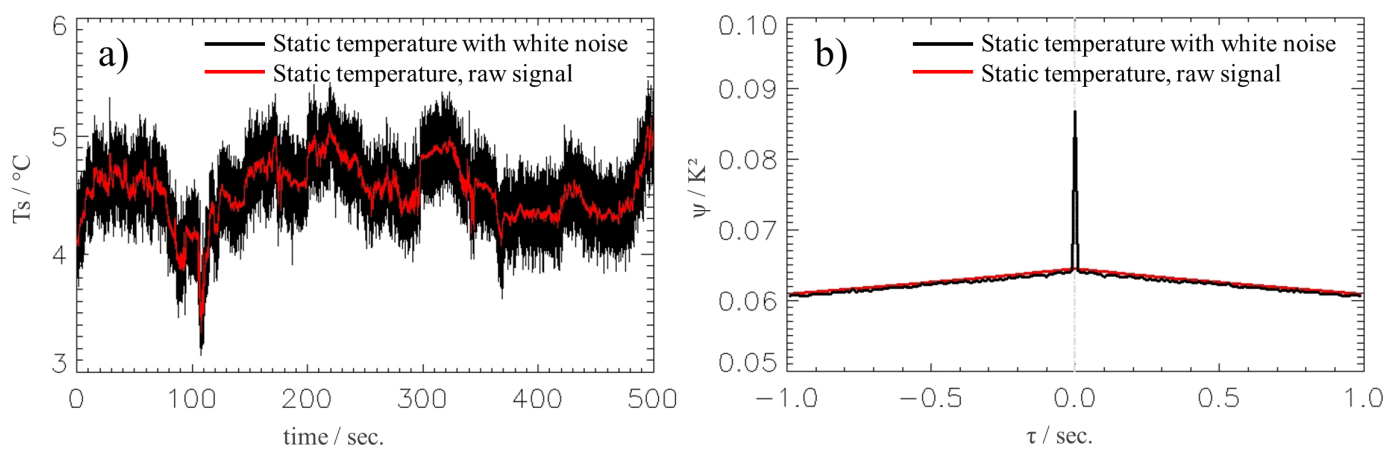

Figure 12. Error analysis for the calculated data caused by measurement uncertainties of the input data for the static temperature. The example in (a) shows a $500 \mathrm{~s}$ time series with and without the artificial white noise contribution. The result of the auto-covariance analysis is presented in (b) with the smooth natural variability of the raw signal and the sharp white noise peak at the time difference $\tau=0$.

to distinguish between correlated atmospheric variations of the original data time series and the uncorrelated white noise error signal. Figure 12a shows an original $500 \mathrm{~s}$ time series of aircraft static temperature data (red line). The observed signal variations are caused by the atmosphere itself and the respective autocovariance clearly proves a correlation between successive data points over many seconds $\left(\Psi_{x x}(\tau) \neq 0\right)$ in Fig. 12b. Note that for an unmodified signal the maximum of an autocovariance function always lies at $\tau=0$, which per definition is the variance of the signal. Adding the white noise error signal to the data leads to a broadening of the original time series (black line). Many of the small-scale atmospheric features seem now to be hidden in the noise. However, Fig. 12b shows that most of the respective autocovariance function looks almost identical to the original one. The atmospheric contributions to the overall variance remain the same with respect to size and temporal behaviour. The only significant difference is the so-called "white noise peak" at $\tau=0$. The height of this peak above the broad atmospheric correlation signal represents the variance caused by noise which is completely uncorrelated between adjacent data points. By simply calculating

$\sigma=\sqrt{\Psi_{x x}(0)-\Psi_{x x}(\delta \tau)}$,

one can easily determine the amount of white noise (the "error") for the investigated constituent. $\delta \tau$ has to be chosen somewhat larger than the width of the white noise peak (i.e. one or two time steps). As one can see, the determination of white noise from an autocovariance function is a fairly simple task. Therefore, it is easy to establish a respective code in the software allowing for a very quick error analysis of the data.

The advantages of the presented error propagation calculation are obvious:

- The method is extremely easy to establish since it concerns only the manipulation of input data and the analysis of processed data, no modification of the data processing algorithms is necessary.
- The method works for any kind of data processing algorithm.

- Changes in the data processing software do not cause any additional work.

- The method does not include any simplifications and can handle even very nonlinear data dependencies.

- The method allows one to study the individual contributions from single sources to the final error by switching all other error signals off.

- The proposed solution can handle any kind of error (statistical as well as systematic).

- The error calculation works very fast and it delivers precise results for every phase of a flight. The measurement uncertainties for flight segments with different meteorological conditions or flight parameters (like aircraft height and speed, mean wind and relative direction to the aircraft) can be estimated separately.

- The method can handle correlated systematic errors by using identical seed values.

However, for statistical reasons the presented error calculation requires a certain time window to calculate a representative autocovariance function. This means that the error analysis for non-stable flight conditions like turns or level changes is not possible.

\subsection{Error discussion}

In the example of Fig. 12 the measurement uncertainty of the static temperature was determined to be $\sigma=0.15 \mathrm{~K}$, showing that it is almost identical to the noise added to the total air temperature signal (as listed in Table 3). For this calculation the measurement uncertainty in form of the white noise signal was added to all the measurement parameters of the system (position and attitude, other avionic data such as $\mathrm{ADC}$ pressure or $\mathrm{ADC}$ temperature and all pressure, flow 
Table 5. List of the calculated measurement uncertainties of the main derived parameters. Details to the method and the calculation of the presented results are given in the text.

\begin{tabular}{llr}
\hline Quantity & Variable & $\sigma$ \\
\hline Static air temperature & $T_{\mathrm{S}}$ & $0.15 \mathrm{~K}$ \\
Humidity mixing ratio & $\mathrm{mr}$ & $2 \%$ \\
& & $\left(4 \%\right.$ below $\left.0.5 \mathrm{~g} \mathrm{~kg}^{-1}\right)$ \\
$3 \% \mathrm{RH}$ \\
Relative humidity & $\mathrm{RH}$ & $\left(5 \%\right.$ RH below $\left.0.5 \mathrm{~g} \mathrm{~kg}^{-1}\right)$ \\
& & $0.35 \mathrm{~K}$ \\
Dewpoint temperature & $T_{\mathrm{d}}$ & $0.25^{\circ}$ \\
Angle of attack & $\alpha$ & $0.25^{\circ}$ \\
Angle of sideslip & $\beta$ & $0.3 \mathrm{~m} \mathrm{~s}^{-1}$ \\
Wind speed & $w_{\mathrm{s}}$ & $2^{\circ}$ \\
Wind angle (at $\left.8 \mathrm{~m} \mathrm{~s}^{-1}\right)$ & $w_{\mathrm{a}}$ & $0.3 \mathrm{~m} \mathrm{~s}^{-1}$ \\
Along-wind component & $u_{\mathrm{f}}$ & $0.3 \mathrm{~m} \mathrm{~s}^{-1}$ \\
Cross-wind component & $v_{\mathrm{f}}$ & $0.25 \mathrm{~m} \mathrm{~s}^{-1}$ \\
Vertical wind & $w$ &
\end{tabular}

angles, temperature or humidity signals of the METPOD). It shows that apart from the total air temperature signal the other parameters have a negligible influence. This is different for the error calculation of wind and humidity as presented in Table 5, where several parameters have a significant contribution to the overall uncertainty. As long as the individual measurement uncertainty is added to all the original time series, the result of the error calculation represents the overall error of the derived quantity. It is difficult to distinguish the individual contribution of single parameters to the overall error, but we will discuss the main error sources for the different wind components below. For this error calculation we analysed the measurement errors for 18 different flight legs during four measurement flights in 2012 and 2013. Two of these flights were performed in the turbulent boundary layer over an Alpine valley and the other two in the vicinity of warm cumulus clouds.

The measurement uncertainties of the humidity in Table 5 for the Ly- $\alpha$ include the overall calibration uncertainty of the instrument as well as the contribution due to the lamp intensity calculation, which was performed as an additional step in the calibration. The measurement uncertainties of the Ly- $\alpha$ are similar to those of the TP3, but due to the faster response time this instrument is the preferred sensor. The measurement uncertainties of the Humicap are almost twice as large.

The calibration error of the flow angles has different sources, the sensor calibration errors and the in-flight calibration errors, as described in Sect. 4.2. The error of the pressure measurement can be added to the raw data immediately, but the in-flight calibration errors have to be accounted for separately. This error contribution must be added directly in the flow angle calculation routine, because it depends on derived quantities and not on raw data. The estimated uncertainties of $\alpha$ and $\beta$ are very similar $\left(\sigma \sim 0.25^{\circ}\right)$, but the error sources of the in-flight calibration are different. The re- sults in Sect. 4.3 demonstrate that just half of the measurement uncertainty of $\alpha$ comes from the $\varepsilon_{\mathbf{b}}$ calculation while the other half is proportional to the magnitude of the value itself (i.e. the relative error). This is different for $\beta$, where the calculation of $\eta_{\mathrm{b}}$ generates the major part of the measurement uncertainty and the relative error is small. This difference has an important implication on the wind accuracy, which primarily depends on both flow angles and the true airspeed. The simplified wind equations given in Eq. (4) demonstrate this relationship. The formulations allow a rough estimation of the expected measurement uncertainties which correspond very well to the calculated values in Table 5. We split the measurement uncertainty for the horizontal wind in the along-wind $\left(\sigma=0.3 \mathrm{~m} \mathrm{~s}^{-1}\right)$ and cross-wind components $\left(\sigma=0.3 \mathrm{~m} \mathrm{~s}^{-1}\right)$, which have the same magnitude but different error sources. The measurement uncertainty of the alongwind component comes primarily from the true airspeed, while the cross-wind component depends on the sideslip angle as one can see from Eq. (4). The measurement uncertainty of the vertical wind component $\left(\sigma=0.25 \mathrm{~m} \mathrm{~s}^{-1}\right)$ is caused by the attack angle calibration. In Sect. 4.3 we estimated three error sources where - depending on the situation - the relative error or the offset error plays the major role. When we look at small attack angles (i.e. $\alpha<2^{\circ}$ ) the relative error is small compared to the $\sigma=0.1^{\circ}$ error from the $\varepsilon_{\mathrm{b}}$ calculation. In this case the error calculation for small attack angles (or also for a mean vertical wind) results in $\sigma<0.2 \mathrm{~m} \mathrm{~s}^{-1}$, while the measurement uncertainty increases significantly for strong vertical wind fluctuations (i.e. $w>5 \mathrm{~m} \mathrm{~s}^{-1}$ or $\alpha> \pm 4^{\circ}$ ) during high-turbulence events. On the other hand the measurement uncertainty of the horizontal wind barely depends on its magnitude. The quality of the measurement uncertainties in Table 5 depends on the accuracy of the measurement uncertainties for the input data. For the parameter in Table 3 we considered all known error sources including the laboratory calibration, the data acquisition or dynamic and temperature effects during flight.

\section{Conclusions}

For high-quality measurements of the 3-D wind with an airborne system, it is crucial to determine the dynamic influences on the measurement equipment during flight. We introduced a method to correct these influences step by step and tested it for the new meteorological sensor system on a Cessna Grand Caravan which is adapted for investigations in the atmospheric boundary layer. The measurement system includes a meteorological sensor package for temperature, humidity and wind, high accuracy position and attitude determination inside the cabin, and a data acquisition system with an integrated time server. The bases for the successful calibration of the system were a well-designed sensor suite, valid laboratory calibrations that are traceable to national standards for all the involved sensors, the opportunity 
to perform a series of test flights during favourable weather situations, and an appropriate software package. This software allows one to perform an automatic and manual quality check on the flight data right after each flight, including the calculation of the flow angle offset $\left(\eta_{\mathrm{b}}\right.$ and $\left.\varepsilon_{\mathrm{b}}\right)$. The calculation is performed for the entire flight, which directly leads to the final measurement data of the meteorological parameters. Even before the calibration procedure in Sect. 4 was completed it was possible to calculate the 3-D wind data and get an estimate of the residual errors. Reverse heading manoeuvres and full circles performed during the test flight were a useful tool to get a qualitative estimate of the wind error.

The correction routines for the temperature and humidity are independent of the specific aircraft, but for the different pressure signals of the gust probe an aircraft has to be tested individually. Four different basic manoeuvres were needed to allow for a stepwise parametrisation of the different dynamic influences on the pressure devices: (i) the static pressure error was calculated from the tower-flyby manoeuvre and with this the static and dynamic pressures were corrected. (ii) The racetrack manoeuvre was performed to check on possible height dependencies of the static pressure error. The test points from these two flight tests were also evaluated for the correction of the attack angle $(\alpha)$. (iii) The constant sideslip manoeuvre was used to calibrate the sideslip angle $(\beta)$. We first evaluated the test points to estimate the $\beta$ dependency of the pressure signal and second calculated the parametrisation of $\beta$ itself. (iv) The last test sequences were harmonic oscillations of the pitch and yaw angles to find any time delays between the different data sources and check on the quality of the wind calculation.

A high accuracy of the attitude angles and especially of the aircraft altitude was the key to the great precision of the calibration. The static pressure error ranges from 1 to $3 \mathrm{hPa}$, depending on the calibrated airspeed with a measurement error of $\sigma=0.1 \mathrm{hPa}$. The parametrisation depends mostly on the dynamic pressure; only a small $\beta$ influence was detected for high deflections. We did not need to correct for any time delays and height or $\alpha$ influences. A linear correction for the flow angle $\alpha$ and $\beta$ was sufficient. For the use of an instrumented pod on this single-engine aircraft, the upwash effects are much stronger compared to the sidewash effect. The pitch and yaw oscillation manoeuvres proved that the calculated wind fluctuations are no longer dependent on the aircraft movements. A big advantage of the stepwise calibration is the possibility to assign the calibration uncertainties to the individual parameters, which allows the determination of the overall measurement uncertainty.
We calculated the error propagation with a new method. The measurement uncertainty is added to the raw data as an artificial white noise signal. The effect can easily be detected at the end of the calculation procedure with an autocovariance analysis. Especially for complex data processing routines with a big number of involved parameters and nonlinear formulations such as humidity and wind calculation, this method displays its strength. We calculated an overall measurement uncertainty for the temperature of $\sigma=0.15 \mathrm{~K}$. The accuracy of the humidity mixing ratio is $\sigma=4$ and $2 \%$ of the measurement value for values below and above $0.5 \mathrm{~g} \mathrm{~kg}^{-1}$, respectively. The error of the wind component along the aircraft is based on the true airspeed error, while for the cross component the $\beta$ error is most important. Both horizontal wind components contribute with the same magnitude to the overall measurement uncertainty which is $\sigma=0.3 \mathrm{~m} \mathrm{~s}^{-1}$. The uncertainty of the vertical wind component is dominated by two major error sources of the attack angle $\alpha$. The first comes from the $\varepsilon_{\mathrm{b}}$ estimation and the second of the linear coefficient for the $\alpha$ calculation which has minor impact on small angles. Thus, the overall measurement uncertainty for the vertical wind is $\sigma=0.25 \mathrm{~m} \mathrm{~s}^{-1}$, which is reduced for small vertical wind fluctuations (e.g. $w<5 \mathrm{~m} \mathrm{~s}^{-1}$ ) to $\sigma<0.2 \mathrm{~m} \mathrm{~s}^{-1}$. To guarantee the high quality of the meteorological data, a regular laboratory calibration of the sensors is necessary. We suggest an annual check based on a short test flight programme to check on the validity of the parametrisation.

We have demonstrated that a vast test program is necessary to calibrate an airborne measurement system. The new system implemented on the Cessna Grand Caravan proved to be a reliable system for high-frequency measurements in the atmospheric boundary layer. The objective determination of the measurement uncertainties builds the basis for any scientific usage of the meteorological data. 


\section{Appendix A: Details for the calculation of the flow angle offsets}

For the calculation of the flow angle offsets described in Sect. 2.2 the vertical wind component of Eq. (1) is needed in the meteorological CS

$w=v_{\mathrm{v}}+\boldsymbol{t a s}_{z}-(\boldsymbol{\Omega} \times \boldsymbol{L})_{z}$

with the aircraft vertical velocity $\left(v_{\mathrm{v}}\right)$, the vertical component of the true airspeed $\left(\boldsymbol{t a s}_{z}\right)$ and the respective vertical component of the motion due to the lever arm $\left(\boldsymbol{\Omega} \times \boldsymbol{L}_{z}\right)$. Note that the signs are changed compared to Eq. (1) due to different definitions of the CS; here $w$ and $v_{\mathrm{v}}$ are positively defined in the upward direction. It is advantageous to use a simplification of Eq. (3)

$\boldsymbol{t a s}_{\mathrm{f}}=\mathrm{TAS} \cdot\left(\begin{array}{c}\cos (\alpha) \cos (\beta) \\ \sin (\beta) \\ \sin (\alpha) \cos (\beta)\end{array}\right)$

as described in earlier references (e.g. Lenschow, 1972). This does not introduce any significant errors, but simplifies the following calculations. The rotation of Eq. (A2) from the aircraft fixed CS (index $f$ ) into the meteorological CS as defined in Sect. 2 leads to

$$
\begin{aligned}
\boldsymbol{t a s}_{z}=\text { TAS } \cdot & (-\cos (\alpha) \cos (\beta) \sin (\Theta) \\
& +\sin (\beta) \sin (\phi) \cos (\Theta) \\
& +\sin (\alpha) \cos (\beta) \cos (\phi) \cos (\Theta)) .
\end{aligned}
$$

It is the only term in Eq. (A1) with a flow angle dependency and, thus, contributing to the derivatives needed in Eq. (7). These are

$$
\begin{aligned}
\frac{\partial w}{\partial \varepsilon_{\mathrm{b}}}=\mathrm{TAS} \cdot( & +\sin (\alpha) \cos (\beta) \sin (\Theta) \\
& +\cos (\alpha) \cos (\beta) \cos (\phi) \cos (\Theta))
\end{aligned}
$$

for the attack angle offset $\left(\varepsilon_{\mathfrak{b}}\right)$ dependency and

$$
\begin{aligned}
\frac{\partial w}{\partial \eta_{\mathrm{b}}}=\mathrm{TAS} \cdot( & +\cos (\alpha) \sin (\beta) \sin (\Theta) \\
& +\cos (\beta) \sin (\phi) \cos (\Theta) \\
& -\sin (\alpha) \sin (\beta) \cos (\phi) \cos (\Theta))
\end{aligned}
$$

for the sideslip angle offset $\left(\eta_{\mathrm{b}}\right)$ dependency. According to Eq. (5), $\varepsilon_{\mathrm{b}}$ and $\eta_{\mathrm{b}}$ are used for the flow angle $(\alpha$ and $\beta$ ) calculation.
During straight flight sections all these angles are small, leading to a value near 1 for the cosines and near 0 for the sines. To obtain Eq. (8) we calculate the average of Eqs. (A4) and (A5). It is clear that the second term on the right-hand side of Eq. (A4) will be dominating because of the cosines. The second term in Eq. (A5) will be small due to the fact that $\sin (\phi)$ changes sign and thus cancels out with the averaging. The other two terms in the equation will be rather small, due to the fact that $\sin (\beta) \ll 1$ under straight flight conditions. Thus, it can be stated that $\left\langle\partial w / \partial \eta_{\mathrm{b}}\right\rangle \ll\left\langle\partial w / \partial \varepsilon_{\mathrm{b}}\right\rangle$.

To fulfill the requirement for Eq. (9), the correlation of $\partial w / \partial \varepsilon_{\mathbf{b}}$ in Eq. (A4) with $\sin (\phi)$ during turns must be small compared to the correlation of $\partial w / \partial \eta_{\mathrm{b}}$ in Eq. (A5) with $\sin (\phi)$. This time the second term in Eq. (A5) is dominating, because it directly contains $\sin (\phi)$. On the other hand, the correlation of $\sin (\phi)$ and $\cos (\phi)$ is small and even vanishes when the same number of left and right turns are flown. Again, the changing sign of the sine function around zero is responsible for this effect. 
Acknowledgements. Providing a research aircraft for meteorological purposes demands the professional support and commitment of the entire team at DLR flight experiments. Therefore, we want to thank the pilots, flight test engineers, technicians and members of operations for their assistance. We thank Ulrich Schumann for useful discussions.

Edited by: T. F. Hanisco

\section{References}

Bange, J., Esposito, M., Lenschow, D. H., Brown, P. R. A., Dreiling, V., Giez, A., Mahrt, L., Malinowki, S. P., Rodi, A. R., Shaw, R. A., Siebert, H., Smit, H., and Zöger, M.: Measurement of Aircraft State and Thermodynamic and Dynamic Variables, in: Airborne Measurements for Environmental Research, edited by: Wendisch, M. and Brenguier, J.-L., 7-75, Wiley-VCH, 2013.

Boegel, W. and Baumann, R.: Test and calibration of the DLR falcon wind measuring system by maneuvers, J. Atmos. Ocean. Tech., 8, 5-18, doi:10.1175/15200426(1991)008<0005:TACOTD>2.0.CO;2, 1991.

Cramer, M.: Performance of IGI AEROcontrol-IId GPS/inertial system, final report, University of Stuttgart, Institute for Photogrammetry, D-70174 Stuttgart, Geschwister-Scholl-Str. 24 D, available at: http://www.ifp.uni-stuttgart.de/publications/2001/ Cramer_Igi01.pdf (last access: 26 January 2015), 2001.

De Leo, R. V. and Hagen, F. W.: Aerodynamic performance of Rosemount model 858AJ air data sensor, Rosemount Report 8767, Aeronautical Research Department, Rosemount Inc., P.O. Box 35129, Minneapolis, Minnesota 55435 USA, 1976.

Druee, C. and Heinemann, G.: A review and practical guide to inflight calibration for aircraft turbulence sensors, J. Atmos. Ocean. Tech., 30, 2820-2837, doi:10.1175/JTECH-D-12-00103.1, 2013.

Giez, A., Zoeger, M., and Dreiling, V.: Processed time sequence measurement data systematic error detection procedure for meteorological or other data uses autocovariance processing of additive white noise, DE 10340793 B4, 2005.

Gracey, W.: Measurement of Aircraft Speed and Altitude, NASA Reference Publication 1046, SpaceAge Control, Inc, 38850 20th Street East Palmdale, CA 93550 USA, available at: www.mecatronica.eesc.usp.br/wiki/upload/1/11/1980_NASA_

Measurement_of_aircraft_airspeed_and_altitude.pdf (last access: 26 January 2015), 1979.

Haering Jr., E. A.: Airdata Calibration of a High-Performance Aircraft for Measuring Atmospheric Wind Profiles, NASA Technical Memorandum 101714, National Aeronautics and Astronautics Administration, NASA, Dryden Flight Research Center, Edwards, California, available at: http://www.nasa.gov/ centers/dryden/pdf/88186main_H-1580.pdf (last access: 26 January 2015), 1990.
Haering Jr., E. A.: Airdata Measurement and Calibration, NASA Technical Memorandum 104316, National Aeronautics and Astronautics Administration, NASA, Dryden Flight Research Center, Edwards, California, available at: http://www.nasa.gov/ centers/dryden/pdf/88377main_H-2044.pdf (last access: 26 January 2015), 1995.

Hauf, T.: Turbulenzmessungen mit dem Forschungsflugzeug Falcon, Meteorol. Rundsch., 37, 163-176, 1984.

Kalogiros, J. A. and Wang, Q.: Aerodynamic effects on wind turbulence measurements with research aircraft, J. Atmos. Ocean. Tech., 19, 1567-1576, doi:10.1175/15200426(2002)019<1567:AEOWTM>2.0.CO;2, 2002.

Khelif, D., Burns, S. P., and Friehe, C. A.: Improved wind measurements on research aircraft, J. Atmos. Ocean. Tech., 16, 860-875, doi:10.1175/1520-0426(1999)016<0860:IWMORA>2.0.CO;2, 1999.

Lenschow, D. H.: The Measurement of Air Velocity and Temperature Using the NCAR Buffalo Aircraft Measuring System, Tech. rep., National Center for Atmospheric Research (NCAR), Boulder, CO, USA, 1972.

Lenschow, D. H.: Probing the atmospheric boundary layer, American Meteorological Society, Boston, MA, USA, 1986.

Lenschow, D. H. and Spyers-Duran, P.: Measurement techniques: air motion sensing, RAF Bulletin 23, NCAR - RAF, NCAR Research Aviation Facility, available at: www.eol.ucar.edu/raf/ Bulletins/bulletin23.html (last access: 26 January 2015), description of air measurement processes at NCAR, 1989.

Liljequist, G. H. and Cehak, K.: Allgemeine Meteorologie, 3rd Edn., Springer Vieweg, 1984.

Mallaun, C. and Giez, A.: The Missing Link: How to optimize pressure calibration using the tower flyby method, in: Proceedings of the 24th Annual SFTE EC Symposium, 2013.

Mayerbuch, I.: Characterization of Humidity Sensors for Application on the High Altitude Research Aircraft HALO, M.S. thesis, Technische Universität München, München, Germany, 2006.

Rodi, A. R. and Leon, D. C.: Correction of static pressure on a research aircraft in accelerated flight using differential pressure measurements, Atmos. Meas. Tech., 5, 2569-2579, doi:10.5194/amt-5-2569-2012, 2012.

Tjernstroem, M. and Friehe, C. A.: Analysis of a radome airmotion system on a twin-jet aircraft for boundary-layer research, J. Atmos. Ocean. Tech., 8, 19-40, doi:10.1175/15200426(1991)008<0019:AOARAM>2.0.CO;2, 1991.

Wilks, D. S.: Statistical Methods in the Atmospheric Sciences, 2nd Edn., Elsevier, 2006. 